\title{
Alternans and spiral breakup in a human ventricular tissue model
}

\author{
K. H. W. J. ten Tusscher and A. V. Panfilov \\ Department of Theoretical Biology, Utrecht University, Utrecht, The Netherlands
}

Submitted 30 January 2006; accepted in final form 5 March 2006

Ten Tusscher, K. H. W. J. and A. V. Panfilov. Alternans and spiral breakup in a human ventricular tissue model. Am J Physiol Heart Circ Physiol 291: H1088-H1100, 2006. First published March 24, 2006; doi:10.1152/ajpheart.00109.2006.-Ventricular fibrillation (VF) is one of the main causes of death in the Western world. According to one hypothesis, the chaotic excitation dynamics during VF are the result of dynamical instabilities in action potential duration (APD) the occurrence of which requires that the slope of the APD restitution curve exceeds 1 . Other factors such as electrotonic coupling and cardiac memory also determine whether these instabilities can develop. In this paper we study the conditions for alternans and spiral breakup in human cardiac tissue. Therefore, we develop a new version of our human ventricular cell model, which is based on recent experimental measurements of human APD restitution and includes a more extensive description of intracellular calcium dynamics. We apply this model to study the conditions for electrical instability in single cells, for reentrant waves in a ring of cells, and for reentry in two-dimensional sheets of ventricular tissue. We show that an important determinant for the onset of instability is the recovery dynamics of the fast sodium current. Slower sodium current recovery leads to longer periods of spiral wave rotation and more gradual conduction velocity restitution, both of which suppress restitution-mediated instability. As a result, maximum restitution slopes considerably exceeding 1 (up to 1.5) may be necessary for electrical instability to occur Although slopes necessary for the onset of instabilities found in our study exceed 1, they are within the range of experimentally measured slopes. Therefore, we conclude that steep APD restitution-mediated instability is a potential mechanism for VF in the human heart.

reentrant arrhythmias; human ventricular myocytes; restitution properties; spiral waves; computer simulation

ONE OF THE MOST extensively investigated hypotheses for ventricular fibrillation (VF) is the so-called restitution hypothesis. In its initial form the hypothesis stated that if the action potential duration (APD) restitution curve has a maximum slope steeper than 1 , it will lead to APD alternans $(16,41)$. In tissue this APD alternans can result in the fragmentation of a spiral wave, leading to fibrillation-like excitation patterns (21, $22,45,48)$. Modeling studies have confirmed that a steep restitution curve indeed promotes instability. However, modeling studies have also shown that the criterion of an APD restitution slope $>1$ is an oversimplification that only holds for very simple models. In more realistic and complex models, it has been shown that other factors such as short-term cardiac memory, electrotonic interactions between cells, conduction velocity $(\mathrm{CV})$ restitution, the range of diastolic intervals (DIs) over which restitution is steeper than 1 , and the range of DIs visited during spiral wave rotation all play an important role in determining whether alternans and spiral breakup will occur $(5$, $7,10,11,42,48,64)$. In an extensive study of restitutioninduced instability, Cherry and Fenton (6), for example,

Address for reprint requests and other correspondence: K. H. W. J. ten Tusscher, Utrecht Univ., Dept. of Theoretical Biology, Padualaan 8, $3584 \mathrm{CH}$ Utrecht, The Netherlands (e-mail: khwjtuss@ hotmail.com). showed that because of strong electrotonic interactions and gradual CV restitution, spiral breakup may not occur even if the APD restitution curve has slope as much as 2 .

The restitution hypothesis has promoted a large number of experimental studies. An important goal of these studies was to measure restitution curves and their slopes. Experiments in animal hearts have shown that the maximum slope of the restitution curve can indeed be $>1$; for example, for pig heart, slopes of 1.29 (27); for dog heart, slopes of 1.13 (25); and for rabbit heart, slopes of 1.3 (18) up to 2.3 (2) were found. However, slopes of $<1$ have also frequently been reported (18, 27). In experiments it was also found that factors other than mere APD restitution slope are important in determining whether alternans or fibrillation occurs. Wu et al. (66) and Banville and Gray (2) showed that whether VF occurred depended both on APD and CV restitution. Banville et al. (1) also demonstrated that cardiac memory effects play a role in whether alternans and VF occur.

Early studies of APD restitution in the human heart suggested that the maximum slope in humans could be close to or slightly larger than 1 (37, 59). More recently, Pak et al. (43) measured human APD restitution in the right ventricular apex and outflow tract. They found that restitution slopes can be considerably steeper than 1 , reporting values of up to 1.28 for the apex and of up to 3.78 for the outflow tract. In addition, they found a positive correlation between arrhythmia inducibility and both steepness and dispersion of the restitution slopes. Yue et al. (67) measured restitution on the left and right ventricular endocardium at 32 sites, reporting restitution slopes varying from site to site and ranging from 0 to 2. The most extensive study on human APD restitution has recently been performed by Nash et al. (38-40). They measured restitution at 256 points covering the complete ventricular epicardium of 14 patients, showing that restitution is distributed heterogeneously but is regionally organized over the epicardial surface, with maximum slopes varying from almost 0 to $>3$, and a mean value of 1.1.

These recent data imply the possibility of steep restitutioninduced instabilities in human cardiac tissue. However, as was mentioned above, the question of whether such instabilities actually do occur also depends on other factors, such as CV restitution, electrotonic interactions and cardiac memory, range of DIs over which the curve is steep, and DIs visited during spiral wave rotation. Because of the substantial limitations to experimental studies involving human cardiac tissue, alternative methods are of great interest. In this study we will investigate the conditions for electrical instability in human ventricular tissue by using the method of mathematical modeling.

For this purpose we developed a new version of our human ventricular cell model (61). The new model is based on recent

\footnotetext{
The costs of publication of this article were defrayed in part by the payment of page charges. The article must therefore be hereby marked "advertisement" in accordance with 18 U.S.C. Section 1734 solely to indicate this fact.
} 
experimental restitution data (39) and incorporates an improved description of intracellular calcium dynamics, by including subspace calcium dynamics that controls L-type calcium current and calcium-induced calcium release (CICR), by modeling CICR with a four-state Markov model for the ryanodine receptor, and by incorporating both fast and slow voltage-gated inactivation of the L-type calcium current. We use our new model to study the onset of alternans in a single cell, for waves propagating in a ring of cardiac tissue (a model for reentry around a fixed path), and for two-dimensional reentry (spiral waves).

In our earlier study, we found that the recovery dynamics of the fast sodium current $\left(I_{\mathrm{Na}}\right)$ are considerably slower than what is often assumed in other modeling studies. $I_{\mathrm{Na}}$ dynamics are an important determinant of CV restitution and of spiral wave rotation speed. Cherry and Fenton (6) have demonstrated that $\mathrm{CV}$ restitution plays an important role in the occurrence of electrical instability, whereas we (63) have previously shown that the speed of spiral wave rotation and hence the DIs visited during spiral wave rotation influence whether alternans instability occurs.

Therefore, we focus on the effect of $I_{\mathrm{Na}}$ recovery dynamics in combination with APD restitution on alternans and spiral breakup. In particular, we use our new model to reproduce a representative range of the experimentally measured restitution curves recently reported by Nash et al. (39), with slopes varying from 0.7 to 2 . We combine these different restitution slopes with three types of $I_{\mathrm{Na}}$ recovery dynamics: slow, medium, and fast recovery. We find that slower $I_{\mathrm{Na}}$ recovery dynamics suppress steep APD restitutionmediated instability and spiral breakup. Under slow $I_{\mathrm{Na}}$ recovery dynamics, maximum restitution slopes significantly steeper than 1 are required to generate electrical instability and breakup. However, such slopes are within the range of restitution slopes reported by Nash et al. (39). Therefore, we conclude that steep restitutionmediated alternans and spiral breakup may exist in the human heart.

\section{MATERIALS AND METHODS}

\section{Model Development}

We developed a new version of our human ventricular cell model. In this new model we included a more extensive description of intracellular calcium handling, incorporating a subsarcolemmal space, describing CICR with a Markov-state model for the ryanodine receptor, including both fast and slow voltage inactivation for the L-type calcium current, and applying some minor changes to parameter values and to slow delayed rectifier time dynamics. Important changes are discussed in detail below. Detailed equations can be found in the APPENDIX; the new default parameter setting of our model can be found in Table 1.

Calcium dynamics. A schematic representation of the calcium dynamics in our new model is given in Fig. 1. The model now contains a description of calcium dynamics in the subspace $\left(\mathrm{Ca}_{\mathrm{ss}}\right)$, cytoplasm $\left(\mathrm{Ca}_{\mathrm{i}}\right)$, and sarcoplasmic reticulum $\left(\mathrm{Ca}_{\mathrm{SR}}\right)$. Calcium in the subspace is buffered, and a diffusion flux is added to allow calcium released in the subspace to flow to the bulk cytoplasm. L-type calcium current and the ryanodine calcium release current now inject calcium into the subsarcolemmal subspace, and, in turn, their dynamics are influenced by the subspace calcium concentration. Sarcolemmal $\mathrm{Ca}^{2+}$ pump current $\left(I_{\mathrm{pCa}}\right)$, background $\mathrm{Ca}^{2+}$ current $\left(I_{\mathrm{bCa}}\right)$, and $\mathrm{Na}^{+} / \mathrm{Ca}^{2+}$ exchanger current $\left(I_{\mathrm{NaCa}}\right)$ still depend on bulk cytoplasmic calcium.

Figure 2 shows a steady-state $1-\mathrm{Hz}$ epicardial action potential for the new version our human ventricular cell model (Fig. $2 A$ ) together with the calcium transients occurring in the subspace (Fig. 2C) and bulk cytoplasm (Fig. $2 B$ ). It can be seen that the calcium transient in the subspace is much larger (peak amplitude of 1.77 vs. $0.0013 \mathrm{mM}$ ), faster (time to peak $\sim 2$ vs. $\sim 35 \mathrm{~ms}$ ), and shorter (duration of $\sim 35 \mathrm{vs}$. $\sim 400 \mathrm{~ms}$ ) than that in the cytoplasm. Maximum upstroke velocity of the action potential is $289 \mathrm{mV} / \mathrm{ms}$, the plateau level is $24.9 \mathrm{mV}$, and resting membrane potential is $-85.8 \mathrm{mV}$, all very similar to the previous version of our model. However, APD at $90 \%$ repolarization $\left(\mathrm{APD}_{90}\right.$ ) now is $306 \mathrm{~ms}$ and was $276 \mathrm{~ms}$ in the previous version of our model. We decided for this change to let our model APD lie more in the midrange of experimentally recorded APDs in single cell [336, 298,360 , and $310 \mathrm{~ms}(28-31)$ ] and tissue preparations [351 and 378 $\mathrm{ms}(8,9,14)]$.

L-type calcium current. L-type calcium current $\left(I_{\mathrm{CaL}}\right)$ (see Eq. 6 of APPENDIX) now injects calcium into the subspace and in turn is inactivated by the $\mathrm{Ca}_{\mathrm{ss}}$ via the inactivation gate $f_{\text {cass }}$ (Fig. 3, $A$ and $B$ ). $I_{\mathrm{CaL}}$ voltage-clamp experiments indicate the presence of both a fast and slow voltage-dependent recovery process $(32,35,46)$. Therefore, we incorporate both a slow voltage inactivation gate $f$ and a fast voltage inactivation gate $f_{2}$, similar to the approach taken by Hund and Rudy (19). Figure $3 C$ shows the steady-state inactivation curves of $f$ and $f_{2}$, and Fig. $3 D$ shows the time constant curves of $f$ and $f_{2}$ together with experimental data. Note that the inactivation rate of $f_{2}$ is slower than that of $f_{\text {cass }}$ but much faster than that of $f$. Note also that as opposed to the $f$ gate, but similar to the $f_{\text {cass }}$ gate, the $f_{2}$ gate inactivates incompletely. These two properties enable the $f_{2}$ gate to take over initial inactivation from $f_{\text {cass, }}$, which is necessary because the short duration of the subspace calcium transient leads to recovery of $f_{\text {cass }}$ during the action potential. In Fig. $3 E$, we show the voltage dependence of our new $I_{\mathrm{CaL}}$ together with experimental data, demonstrating a good agreement between model and experiment (34).

CICR current. In our new model, we use a reduced version of the Markov-state ryanodine receptor model developed by Shannon et al. (54) and Stern et al. (57) to describe CICR. Figure $4 A$ shows the original four-state Markov model of the ryanodine receptor developed by Shannon et al. (54) and Stern et al. (57). It can be seen that the model incorporates both the influence of subspace calcium concentration (the trigger) and sarcoplasmic reticulum calcium concentration (the load) on receptor opening and closing dynamics by making transition rates depend on these concentrations.

For computational efficiency, we reduced the dimensionality of the Markov model by using quasi-steady-state assumptions to the following set of equations

$$
\begin{aligned}
\frac{\mathrm{d} \overline{\mathrm{R}}}{\mathrm{d} t} & =-k_{2} \mathrm{Ca}_{\mathrm{SS}} \overline{\mathrm{R}}+k_{4}(1-\overline{\mathrm{R}}) \\
\mathrm{O} & =\frac{k_{1} \mathrm{Ca}_{S S}^{2} \overline{\mathrm{R}}}{k_{3}+k_{1} \mathrm{Ca}_{\mathrm{SS}}^{2}} \\
I_{\text {rel }} & =V_{\text {rel }} \mathrm{O}\left(\mathrm{Ca}_{\mathrm{SR}}-\mathrm{Ca}_{\mathrm{SS}}\right)
\end{aligned}
$$

where $\overline{\mathrm{R}}=\mathrm{R}+\mathrm{O}$, with $\mathrm{R}$ the resting closed state and $\mathrm{O}$ the open conducting state; and where $I_{\text {rel }}$ is the CICR current.

We determined the gain function for the dependence of calcium release on SR calcium load. (Gain is defined as the CICR flux normalized by the L-type calcium current flux triggering it.) Figure $4 B$ shows gain in our model as a function of free calcium in the sarcoplasmic reticulum. For qualitative comparison, experimental data from Shannon et al. (53) in rabbit ventricular myocytes are added; unfortunately we could not find data on the gain function in human ventricular myocytes. We can see that our model generates a nonlinear response function, as is the case for the experimental data.

\section{Numerical Methods}

Action potential generation in single cells was described using the following differential equation (23) 
Table 1. Default model parameter settings

\begin{tabular}{|c|c|c|}
\hline Parameter & Definition & Value \\
\hline$R$ & Gas constant & $8.3143 \mathrm{~J} \cdot \mathrm{K}^{-1} \cdot \mathrm{mol}^{-1}$ \\
\hline$T$ & Temperature & $310 \mathrm{~K}$ \\
\hline$F$ & Faraday constant & $96.4867 \mathrm{C} / \mathrm{mmol}$ \\
\hline$C_{\mathrm{m}}$ & Cell capacitance per unit surface area & $2.0 \mu \mathrm{F} / \mathrm{cm}^{2}$ \\
\hline $\mathrm{S}$ & Surface to volume ratio & $0.2 \mu \mathrm{m}^{-1}$ \\
\hline$\rho$ & Cellular resistivity & $162 \Omega \cdot \mathrm{cm}$ \\
\hline $\mathrm{V}_{\mathrm{c}}$ & Cytoplasmic volume & $16.404 \mu \mathrm{m}^{3}$ \\
\hline $\mathrm{V}_{\mathrm{sr}}$ & Sarcoplasmic reticulum volume & $1.094 \mu \mathrm{m}^{3}$ \\
\hline $\mathrm{V}_{\mathrm{ss}}$ & Subspace volume & $0.05468 \mu \mathrm{m}^{3}$ \\
\hline $\mathrm{K}_{\mathrm{o}}$ & Extracellular $\mathrm{K}^{+}$concentration & $5.4 \mathrm{mM}$ \\
\hline $\mathrm{Na}_{\mathrm{o}}$ & Extracellular $\mathrm{Na}^{+}$concentration & $140 \mathrm{mM}$ \\
\hline $\mathrm{Ca}_{\mathrm{o}}$ & Extracellular $\mathrm{Ca}^{2+}$ concentration & $2 \mathrm{mM}$ \\
\hline$G_{\mathrm{Na}}$ & Maximal $I_{\mathrm{Na}}$ conductance & $14.838 \mathrm{nS} / \mathrm{pF}$ \\
\hline$G_{\mathrm{K} 1}$ & Maximal $I_{\mathrm{K} 1}$ conductance & $5.405 \mathrm{nS} / \mathrm{pF}$ \\
\hline$G_{\mathrm{to}}$, epi, M & Epicardial $I_{\text {to }}$ conductance & $0.294 \mathrm{nS} / \mathrm{pF}$ \\
\hline$G_{\mathrm{to}}$, endo & Maximal endocardial $I_{\text {to }}$ conductance & $0.073 \mathrm{nS} / \mathrm{pF}$ \\
\hline$G_{\mathrm{Kr}}$ & Maximal $I_{\mathrm{Kr}}$ conductance & $0.153 \mathrm{nS} / \mathrm{pF}$ \\
\hline$G_{\mathrm{Ks}}$, epi, endo & Maximal epi-and endocardial $I_{\mathrm{Ks}}$ conductance & $0.392 \mathrm{nS} / \mathrm{pF}$ \\
\hline$G_{\mathrm{Ks}}, \mathrm{M}$ & Maximal $\mathrm{M}$ cell $I_{\mathrm{Ks}}$ conductance & $0.098 \mathrm{nS} / \mathrm{pF}$ \\
\hline$p_{\mathrm{KNa}}$ & Relative $I_{\mathrm{Ks}}$ permeability to $\mathrm{Na}^{+}$ & 0.03 \\
\hline$G_{\mathrm{CaL}}$ & Maximal $I_{\mathrm{CaL}}$ conductance & $3.980^{-5} \mathrm{~cm} \cdot \mathrm{ms}^{-1} \cdot \mu \mathrm{F}^{-1}$ \\
\hline$k_{\mathrm{NaCa}}$ & Maximal $I_{\mathrm{NaCa}}$ & $1,000 \mathrm{pA} / \mathrm{pF}$ \\
\hline$\gamma$ & Voltage dependence parameter of $I_{\mathrm{NaCa}}$ & 0.35 \\
\hline$K_{\mathrm{mCa}}$ & $\mathrm{Ca}_{\mathrm{i}}$ half-saturation constant for $I_{\mathrm{NaCa}}$ & $1.38 \mathrm{mM}$ \\
\hline$K_{\text {mNai }}$ & $\mathrm{Na}_{\mathrm{i}}$ half-saturation constant for $I_{\mathrm{NaCa}}$ & $87.5 \mathrm{mM}$ \\
\hline$k_{\text {sat }}$ & Saturation factor for $I_{\mathrm{NaCa}}$ & 0.1 \\
\hline$\alpha$ & Factor enhancing outward nature of $I_{\mathrm{NaCa}}$ & 2.5 \\
\hline$P_{\mathrm{NaK}}$ & Maximal $I_{\mathrm{NaK}}$ & $2.724 \mathrm{pA} / \mathrm{pF}$ \\
\hline$K_{\mathrm{mK}}$ & $\mathrm{K}_{\mathrm{o}}$ half-saturation constant of $I_{\mathrm{NaK}}$ & $1 \mathrm{mM}$ \\
\hline$K_{\mathrm{mNa}}$ & $\mathrm{Na}_{\mathrm{i}}$ half-saturation constant of $I_{\mathrm{NaK}}$ & $40 \mathrm{mM}$ \\
\hline$G_{\mathrm{pK}}$ & Maximal $I_{\mathrm{pK}}$ conductance & $0.0146 \mathrm{nS} / \mathrm{pF}$ \\
\hline$G_{\mathrm{pCa}}$ & Maximal $I_{\mathrm{pCa}}$ conductance & $0.1238 \mathrm{nS} / \mathrm{pF}$ \\
\hline$K_{\mathrm{pCa}}$ & Half-saturation constant of $I_{\mathrm{pCa}}$ & $0.0005 \mathrm{mM}$ \\
\hline$G_{\mathrm{bNa}}$ & Maximal $I_{\mathrm{bNa}}$ conductance & $0.000290 \mathrm{nS} / \mathrm{pF}$ \\
\hline$G_{\mathrm{bCa}}$ & Maximal $I_{\mathrm{bCa}}$ conductance & $0.000592 \mathrm{nS} / \mathrm{pF}$ \\
\hline$V_{\operatorname{maxup}}$ & Maximal $I_{\text {up }}$ conductance & $0.006375 \mathrm{mM} / \mathrm{ms}$ \\
\hline$K_{\text {up }}$ & Half-saturation constant of $I_{\text {up }}$ & $0.00025 \mathrm{mM}$ \\
\hline$V_{\text {rel }}$ & Maximal $I_{\text {rel }}$ conductance & $40.8 \mathrm{mM} / \mathrm{ms}$ \\
\hline$k_{1}^{\prime}$ & $\mathrm{R}$ to $\mathrm{O}$ and RI to I $I_{\text {rel }}$ transition rate & $0.15 \mathrm{mM}^{-2} \cdot \mathrm{ms}^{-1}$ \\
\hline$k_{2}^{\prime}$ & $\mathrm{O}$ to I and R to RI $I_{\text {rel }}$ transition rate & $0.045 \mathrm{mM}^{-1} \cdot \mathrm{ms}^{-1}$ \\
\hline$k_{3}$ & $\mathrm{O}$ to $\mathrm{R}$ and I to RI $I_{\text {rel }}$ transition rate & $0.060 \mathrm{~ms}^{-1}$ \\
\hline$k_{4}$ & I to $O$ and RI to I $I_{\text {rel }}$ transition rate & $0.000015 \mathrm{~ms}^{-1}$ \\
\hline $\mathrm{EC}$ & CaSR half-saturation constant of $k_{\text {casr }}$ & $1.5 \mathrm{mM}$ \\
\hline $\max _{\mathrm{sr}}$ & Maximum value of $k_{\text {casr }}$ & 2.5 (dimensionless) \\
\hline $\min _{\text {sr }}$ & Minimum value of $k_{\text {casr }}$ & 1 (dimensionless) \\
\hline$V_{\text {leak }}$ & Maximal $I_{\text {leak }}$ conductance & $0.00036 \mathrm{mM} / \mathrm{ms}$ \\
\hline$V_{\text {xfer }}$ & Maximal $I_{\text {xfer }}$ conductance & $0.0038 \mathrm{mM} / \mathrm{ms}$ \\
\hline $\mathrm{Buf}_{\mathrm{c}}$ & Total cytoplasmic buffer concentration & $0.2 \mathrm{mM}$ \\
\hline$K_{\text {bufc }}$ & $\mathrm{Ca}_{\mathrm{i}}$ half-saturation constant for cytoplasmic buffer & $0.001 \mathrm{mM}$ \\
\hline $\mathrm{Buf}_{\mathrm{sr}}$ & Total sarcoplasmic buffer concentration & $10 \mathrm{mM}$ \\
\hline$K_{\text {bufsr }}$ & $\mathrm{Ca}_{\mathrm{SR}}$ half-saturation constant for sarcoplasmic buffer & $0.3 \mathrm{mM}$ \\
\hline $\mathrm{Buf}_{\mathrm{ss}}$ & Total subspace buffer concentration & $0.4 \mathrm{mM}$ \\
\hline$K_{\text {bufss }}$ & Cass half-saturation constant for subspace buffer & $0.00025 \mathrm{mM}$ \\
\hline
\end{tabular}

$I_{\mathrm{Na}}, \mathrm{Na}^{+}$current; $I_{\mathrm{K} 1}$, inward rectifier $\mathrm{K}^{+}$current; $I_{\mathrm{to}}$, transient outward current, $I_{\mathrm{Kr}}$, rapid delayed rectifier current; $I_{\mathrm{Ks}}$, slow delayed rectifier current; $I_{\mathrm{CaL}}$, L-type $\mathrm{Ca}^{2+}$ current; $I_{\mathrm{NaCa}}, \mathrm{Na}^{+} / \mathrm{Ca}^{2+}$ exchanger current; $I_{\mathrm{NaK}}, \mathrm{Na}^{+}-\mathrm{K}^{+}$pump current; $I_{\mathrm{bNa}}$, background $\mathrm{Na}^{+}$current; $I_{\mathrm{bCa}}$, background $\mathrm{Ca}^{2+}$ current; $I_{\mathrm{pK}}$, plateau $\mathrm{K}^{+}$current; $I_{\mathrm{pCa}}$, sarcolemmal Ca ${ }^{2+}$ pump current; $I_{\text {rel }}$, calcium-induced calcium release current; $I_{\text {up }}$, sarcoplasmic reticulum (SR) $\mathrm{Ca}^{2+}$ pump current; $I_{\text {leak }}, \mathrm{SR}$ $\mathrm{Ca}^{2+}$ leak current; $I_{\text {xfer }}$, diffusive $\mathrm{Ca}^{2+}$ current between diadic subspace and bulk cytoplasm; O, open conducting state of $I_{\text {rel }}$; R, resting closed state of $I_{\text {rel }} ; \mathrm{I}$, inactivated closed state of $I_{\mathrm{rel}}$; RI, resting inactivated closed state of $I_{\mathrm{rel}}$

$$
C_{\mathrm{m}} \frac{\mathrm{d} V}{\mathrm{~d} t}=-\left(I_{\text {ion }}+I_{\text {stim }}\right)
$$

where $C_{\mathrm{m}}$ is the membrane capacitance, $V$ is the transmembrane potential, $I_{\text {stim }}$ is the externally applied transmembrane current, and $I_{\text {ion }}$ is the sum of all transmembrane ionic currents. For $I_{\text {ion }}$, we use our new version of the human ventricular cell model.

Similarly, action potential generation and propagation in onedimensional cables and rings and two-dimensional sheets of cardiac tissue were described by using the following parabolic reaction diffusion equation (23)

$$
C_{\mathrm{m}} \frac{\partial V}{\partial t}=-\left(I_{\text {ion }}+I_{\text {stim }}\right)+\nabla D \nabla V
$$

where $D$ is a tensor describing the conductivity of the tissue and $\nabla$ is the one- or two-dimensional gradient operator. In one dimension, $D=$ $0.00154 \mathrm{~cm}^{2} / \mathrm{ms}$; in two dimensions, $D_{i j}=0.00154 \mathrm{~cm}^{2} / \mathrm{ms}$ for $i=j$, and $D_{i j}=0 \mathrm{~cm}^{2} / \mathrm{ms}$ for $i \neq j$. With these values we obtain a maximum 


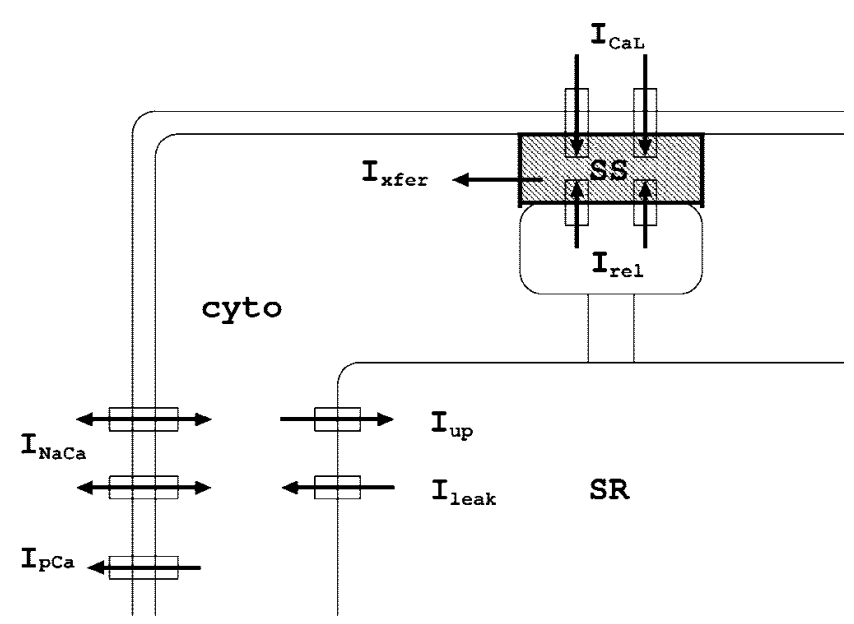

Fig. 1. Calcium compartments involved in excitation-contraction coupling. L-type calcium channels $\left(I_{\mathrm{CaL}}\right)$ release calcium in the diadic space or subspace (SS), where sarcolemmal membrane and membrane of the sarcoplasmic reticulum $(\mathrm{SR})$ are in close proximity. Ryanodine receptors $\left(I_{\mathrm{rel}}\right)$ sense this elevation of calcium in the subspace and respond with a release of calcium from the SR. Through diffusion $\left(I_{\mathrm{xfer}}\right)$, the calcium released in the subspace travels to the cytoplasm (cyto). Sodium-calcium exchangers $\left(I_{\mathrm{NaCa}}\right)$ and the sarcolemmal calcium pump $\left(I_{\mathrm{pCa}}\right)$ pump calcium out of the cytoplasm to the exterior of the cell. Calcium pumps in the membrane of the SR $\left(I_{\mathrm{up}}\right)$ pump calcium out of the cytoplasm back into the SR. A small leakage current $\left(I_{\text {leak }}\right)$ leaks calcium from the SR to the cytoplasm.

planar $\mathrm{CV}$ of $68 \mathrm{~cm} / \mathrm{s}$, which is the velocity found for conductance along the fiber direction in human myocardium (60).

Physical units used in our model are as follows, time $(t)$ in milliseconds, voltage $(V)$ in millivolts, current densities $\left(I_{X}\right)$ in picoamperes per picofarad, and ionic concentrations $\left(X_{i}, X_{o}\right)$ in millimoles per liter.

For single cell simulations, forward Euler integration with a time step of $\Delta t=0.02 \mathrm{~ms}$ was used to integrate $E q$. 4. For one- and two-dimensional computations, the forward Euler method was used to integrate $E q .5$ with a space step of $\Delta x=0.25 \mathrm{~mm}$ and a time step of $\Delta t=0.02 \mathrm{~ms}$. In all cases, the Rush and Larsen integration scheme (52) was used to integrate the Hodgkin-Huxley type equations for the gating variables of the various time-dependent currents $(m, h$, and $j$ for $I_{\mathrm{Na}} ; r$ and $s$ for $I_{\mathrm{to}} ; x_{\mathrm{r} 1}$ and $x_{\mathrm{r} 2}$ for $I_{\mathrm{Kr}} ; x_{\mathrm{s}}$ for $I_{\mathrm{Ks}} ;$ and $d, f, f_{2}$, and $f_{\text {cass }}$ for $\left.I_{\mathrm{CaL}}\right)$.

We measure APD at either $50 \%$ or $90 \%$ repolarization and will refer to these values as $\mathrm{APD}_{50}$ and $\mathrm{APD}_{90}$, respectively. $\mathrm{APD}_{90}$ values will be used, unless stated otherwise. $\mathrm{APD}_{50}$ values are used to allow comparison between experimental activation recovery interval (ARI) data obtained by Nash et al. (38-40). It has been shown that ARI (measured as the interval from the minimum $\mathrm{d} V / \mathrm{d} t$ during the QRS wave to the maximum $\mathrm{d} V / \mathrm{d} t$ of the T-wave) typically corresponds with APD of $\sim 50 \%$ repolarization (17).

In single cells, we apply both the standard S1-S2 and the dynamic protocols to determine APD restitution. For the S1-S2 protocol, $10 \mathrm{~S} 1$ stimuli are applied at a specified basic cycle length (BCL) followed by a single S2 extrastimulus delivered at some DI after the action potential generated by the last S1 stimulus. An APD restitution curve is generated by decreasing DI and plotting the APDs generated by the S2 stimuli against the preceding DIs. In this study, we use $\mathrm{APD}_{50}$ to allow comparison with the experimental studies of Nash et al. (39). For the dynamic restitution protocol, a series of 50 stimuli is applied at a specified BCL, after which cycle length is decreased. The APD restitution curve is obtained by plotting the final APD for each BCL against the final DI.

In cables we apply the dynamic restitution protocol to determine both dynamic APD restitution and CV restitution. We do so by pacing one end of the 800-cell-long cable at a certain BCL until a steady-state APD and CV are reached, after which the cycle length is decreased. In rings, APD and CV restitution can be obtained in the absence of external pacing. Here the restitution protocol is started by applying a single external stimulus generating a wave that is allowed to propagate only in one direction and that will result in the repeated rotation of a wave along the ring. The cycle length of excitation is determined by the ring length. APD and CV can be determined as a function of ring length and hence cycle length and DI. We start with a ring of 1,400 cells $(35 \mathrm{~cm})$ and stepwise reduce its length to decrease cycle length and hence obtain a restitution curve.

We use two-dimensional tissue sheets of $1,000 \times 1,000$ cells (space step $\Delta x=0.25 \mathrm{~mm}$ ). In two dimensions, spiral waves are generated by first applying an S1 stimulus producing a planar wavefront propagating in one direction; then, when the refractory tail of this wave crosses the middle of the medium, an S2 stimulus is applied, generating a second wavefront perpendicular to the first. This produces a second wavefront with a free end around which it curls, forming a spiral wave. Stimulus currents lasted for 2 (S1) and 5 (S2) $\mathrm{ms}$ and were twice the diastolic threshold.

Single-cell, cable, and ring simulations were coded in $\mathrm{C}++$ and run on a single processor of a Dell 650 Precision Workstation (dual Intel xeon $2.66 \mathrm{GHz}$ ). Two-dimensional simulations were coded in $\mathrm{C}++$ and MPI and were run on 20 processors of a Beowulf cluster consisting of 14 Dell 650 Precision Workstations (dual Intel xeon 2.66 $\mathrm{GHz})$.

\section{RESULTS}

\section{APD Restitution Properties of Human Ventricular Cell Model}

Our new model reproduces a representative range of restitution curves recently reported by Nash et al. (38-40). Figure $5, A-D$, shows restitution curves for four different parameter settings of our model together with four different experimental restitution curves of Nash et al. Experimental and model restitution curves were obtained using an S1-S2 protocol at a BCL of $600 \mathrm{~ms}$. In experiments, ARIs were measured, which correspond to $\mathrm{APD}_{50}$ (see METHODS). Model restitution curves are plotted for both $\mathrm{APD}_{50}$ and $\mathrm{APD}_{90}$ levels. Note that there is good agreement between experimental and model curves for
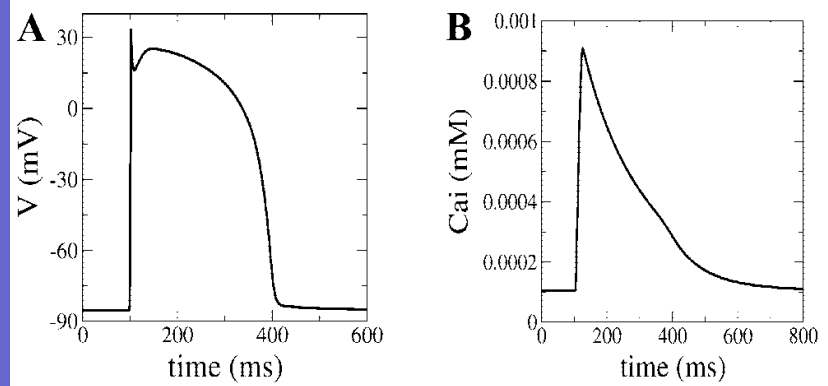

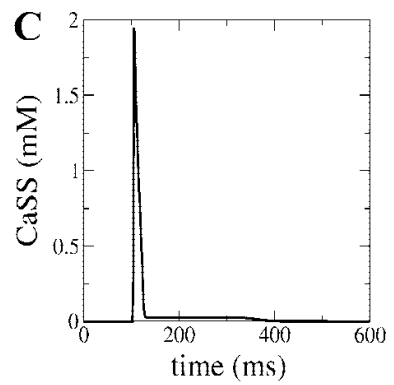

Fig. 2. $A$ : steady-state epicardial action potential $(V)$ shape at $1-\mathrm{Hz}$ pacing. $B$ : steadystate cytoplasmic calcium $\left(\mathrm{Ca}_{\mathrm{i}}\right)$ transient at $1-\mathrm{Hz}$ pacing. $C$ : steady-state subspace calcium $\left(\mathrm{Ca}_{\mathrm{ss}}\right)$ transient at $1-\mathrm{Hz}$ pacing. 

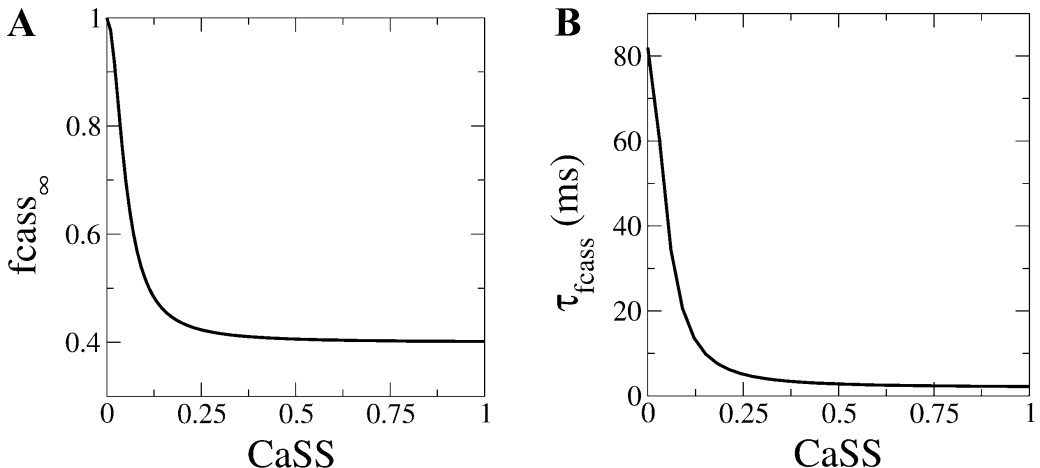

Fig. 3. A: steady-state subspace calcium inactivation $\left(f_{\text {cass }}\right)$ curve. $B$ : time constant curve of subspace calcium inactivation $\left(\tau_{\text {fcass }}\right) . C$ : steady-state curves of slow $\left(f_{\infty}\right)$ and fast $\left(f_{2 \infty}\right)$ voltage inactivation. $D$ : time constant curves of slow $\left(\tau_{\mathrm{f}}\right)$ and fast $\left(\tau_{\mathrm{f} 2}\right)$ voltage inactivation. Experimental (exp) time constants are added for comparison and are taken from Beuckelmann et al (5), Benitah et al. (3), Mewes et al. (36), Sun et al. (58), Li et al. (32), Pelzmann et al. (46), and Magyar et al. (35). Experimental inactivation time constants represent the slowest time scale of calcium current inactivation, thus corresponding to $f$ gate inactivation. For recovery from inactivation, both fast and slow experimental time constants are available, corresponding to $f_{2}$ and $f$ gate recovery. $E$ : peak current-voltage relationship of the L-type calcium current as determined in simulated voltageclamp experiments. Experimental data are from Magyar et al. (34).
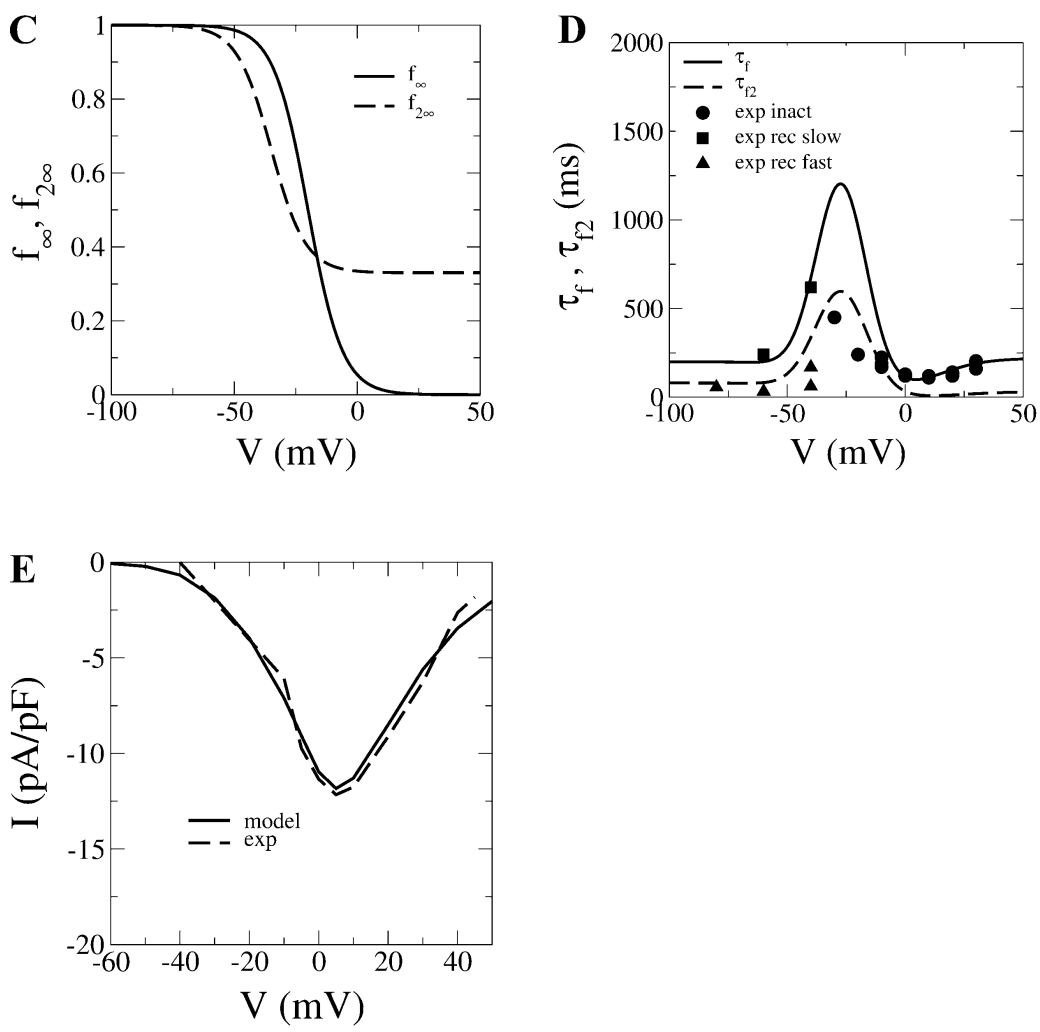

DIs of $200 \mathrm{~ms}$ and smaller, i.e., for DIs that determine the maximum slope of the restitution curve and that determine the onset of instability. For this study we use four parameter settings corresponding to four different slopes of the restitution curves. For setting 1 , the maximum restitution slope $\left(S_{\max }\right)$ is 0.7 (Fig. 5A); for setting 2, it is 1.1 (Fig. $5 B$ ); for setting 3, it is 1.4 (Fig. $5 C$ ); and for setting 4 , it is 1.9 (Fig. $5 D$ ). Table 1 lists the complete default parameter setting, and Table 2 lists
Fig. 4. A: schematic drawing of the 4-state ryanodine receptor Markov model of Shannon et al. (54) and Stern et al. (57). O, open conducting state; $\mathrm{R}$, resting closed state; I, inactivated closed state; $\mathrm{RI}$, resting inactivated closed state. $k_{3}$ and $k_{4}$ are constants; the values of $k_{1}$ and $k_{2}$ are dependent on SR calcium concentration: opening rate is sped up and closing rate slowed down by higher SR calcium load. In addition, $k_{1}$ is multiplied by the square of the subspace calcium concentration, and $k_{2}$ is multiplied by the subspace calcium concentration to give a final rate constant: opening and closing rate increase with triggering subspace calcium levels, but opening more so than closing. $B$ : nonlinear gain function of calcium-induced calcium release (CICR) as a function of free calcium concentration in the sarcoplasmic reticulum $\left(\mathrm{Ca}_{\mathrm{SR}}\right)$. Experimental data from Shannon et al. (53) for rabbit ventricular myocytes are added for comparison.
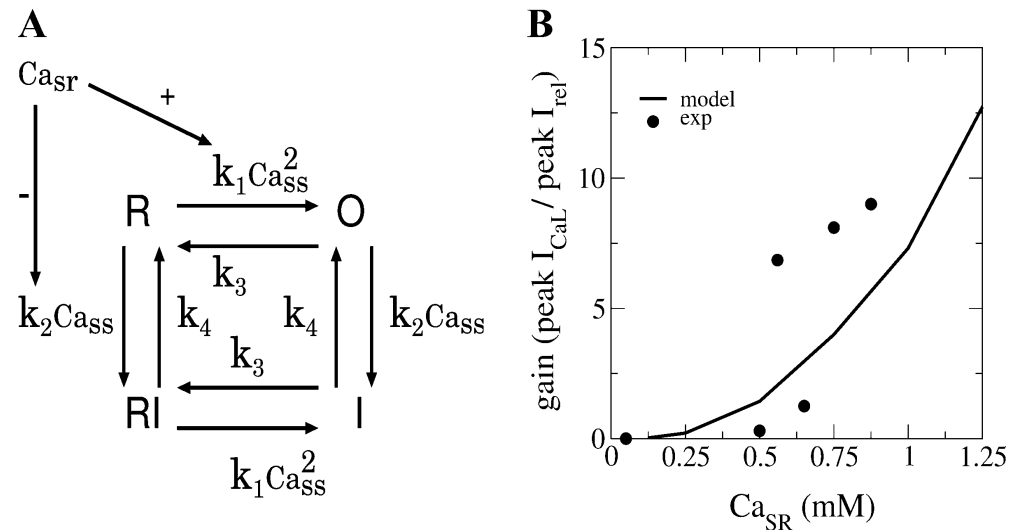

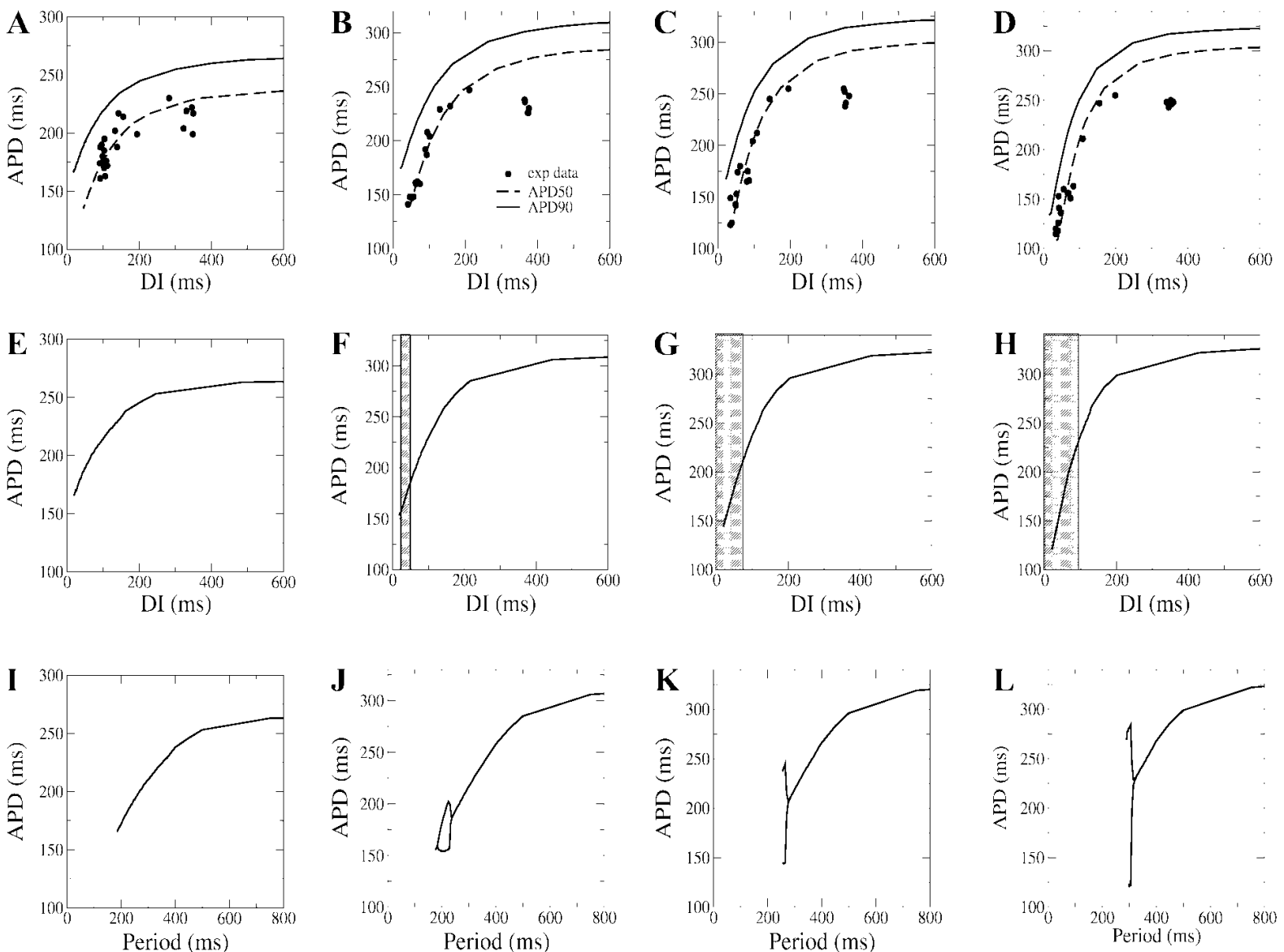

Fig. 5. Single-cell action potential duration (APD) restitution. A-D: restitution curves obtained using a S1-S2 restitution protocol for a basic cycle length (BCL) of $600 \mathrm{~ms}$ measuring APD at 50\% (APD (A) and 90\% repolarization (APD 90 ) for 4 different parameter settings of our model (Table 2). APD is plotted against diastolic interval (DI). Experimental activation recovery interval (ARI) restitution curves (exp) are from Nash et al. (38-40). $E-H$ : restitution curves obtained using a dynamic restitution protocol measuring $\mathrm{APD}_{90}$ for the 4 same parameter settings. APD is plotted against DI. Gray shading is used to indicate the region of the restitution curve where the slope exceeds 1 . I-L: same dynamic restitution curves as in $E-H$, but now plotting APD against stimulation period. Splitting of the restitution curve indicates the presence of 2 APDs for a single period: APD alternans.

the parameter values varied to obtain these different restitution slopes.

\section{Conditions for Alternans in Human Ventricular Tissue}

Restitution and alternans in single cells. It has been suggested by Koller et al. (25) that the slope of the dynamic restitution curve is a better predictor for alternans instability than that of the S1-S2 restitution curve. Therefore we also

Table 2. Four different model parameter settings

\begin{tabular}{cccccc}
\hline \hline Parameter Setting & $G_{\mathrm{Kr}}$ & $G_{\mathrm{Ks}}$ & $G_{\mathrm{pCa}}$ & $G_{\mathrm{pK}}$ & $\tau_{f}$ inact \\
\hline Slope 0.7 & 0.134 & 0.270 & 0.0619 & 0.0730 & $\times 0.6$ \\
Slope 1.1 & 0.153 & 0.392 & 0.1238 & 0.0146 & normal \\
Slope 1.4 & 0.172 & 0.441 & 0.3714 & 0.0073 & $\times 1.5$ \\
Slope 1.8 & 0.172 & 0.441 & 0.8666 & 0.00219 & $\times 2$ \\
\hline
\end{tabular}

Parameter values for the setting with a restitution slope of 1.1 correspond to the values in Table 1. Parameters that were varied were maximum conductance of the $I_{\mathrm{Kr}}, I_{\mathrm{Ks}}, I_{\mathrm{pCa}}$, and $I_{\mathrm{pK}}$ currents (all in $\mathrm{nS} / \mathrm{pF}$ ). An additional parameter that was used was the time constant $\left(\tau_{f}\right)$ for the $f$ gate. For the slope 1.1 setting, the time constant was as given in Fig. $3 D$; for the other settings the time constant was multiplied by a factor (given in right column) for the voltage range $V>$ $0 \mathrm{mV}$, thus rescaling inactivation but not recovery kinetics $\left(\tau_{f}\right.$ inact). determined dynamic restitution for our four different model settings. Figure $5, E-H$, shows restitution curves obtained using a dynamic restitution protocol and measuring $\mathrm{APD}_{90}$. Maximum restitution slopes for our four setting are now 0.8 (Fig. 5E), 1.1 (Fig. 5F), 1.4 (Fig. 5G), and 1.8 (Fig. 5H), respectively. This agrees with experimental data from Pak et al. (43), who demonstrated similar maximum slopes for S1-S2 and dynamic restitution in the human ventricles.

In Fig. 5, $I-L$, we show APD restitution for the same dynamic restitution protocol but now with APD plotted vs. period. We can see that for setting 1 (Fig. 5I), no alternans occurs. For setting 2 (Fig. 5J), APD alternans occurs, resulting in the splitting of the restitution curve in an upper and lower arm representing the long and short action potentials. Alternans occurs for periods between 236 and $182 \mathrm{~ms}$, corresponding to DIs between 51 and $24 \mathrm{~ms}$ for which the slope of the restitution curve in Fig. $5 F$ exceeds 1 . Alternans reaches a maximum amplitude of $48 \mathrm{~ms}$ at a period of $226 \mathrm{~ms}$ and then decreases in amplitude and disappears for periods $<182 \mathrm{~ms}$, qualitatively similar to what occurs in the model of Fox et al. (12). For setting 3 (Fig. $5 K$ ), APD alternans occurs for periods $<280 \mathrm{~ms}$, corresponding to DIs $<74 \mathrm{~ms}$, for which restitution slope in 
Fig. $5 G$ is $>1$, and reaches a maximum amplitude of $100 \mathrm{~ms}$. For setting 4 (Fig. $5 L$ ), alternans occurs for BCL of $320 \mathrm{~ms}$ and smaller, corresponding to DIs $<94 \mathrm{~ms}$, for which slope in Fig. $5 H$ is $>1$, and reaches a maximum amplitude of $162 \mathrm{~ms}$. Our finding that alternans amplitude increases with restitution slopes is consistent with experimental results $(24,26)$.

Restitution and alternans in a ring. The occurrence of alternans in a single cell does not necessarily imply that alternans and spiral breakup occurs in tissue. In tissue, additional factors besides APD restitution, such as electrotonic interactions and CV restitution, the range of DIs for which restitution is steep, and the range of DIs visited during spiral wave rotation, all play a role in determining whether alternans occurs $(5-7,10,11,42,48,64)$.

In our previous study (61), we found that recovery of inactivation of human cardiac fast sodium channels is considerably slower than that of the phase-one Luo-Rudy model (33), which is used in many cardiac tissue models. $I_{\mathrm{Na}}$ dynamics may effect period of spiral wave rotation, $\mathrm{CV}$ restitution, and spiral wave meander pattern, all of which are known to affect the occurrence of alternans instability. Therefore, in tissue simulations, we investigate the combined effect of APD restitution and $I_{\mathrm{Na}}$ recovery dynamics on dynamical instability. To do so, we considered three different descriptions for the fast $I_{\mathrm{Na}}$ dynamics: the default formulation of our model (61) (referred to as standard $I_{\mathrm{Na}}$ setting); an $I_{\mathrm{Na}}$ formulation using $m, h$, and $j$ gate dynamics as in the phase-one Luo-Rudy model (33) (referred to as the LR $I_{\mathrm{Na}}$ setting); and an intermediate setting. The intermediate setting is similar to the standard $I_{\mathrm{Na}}$ setting, except that $\tau_{j \text {,intermediate }}=0.5 \times \tau_{j, \text { standard. }}$. Note that changes in our model's fast $I_{\mathrm{Na}}$ dynamics have virtually no effect on the single-cell APD restitution properties shown in Fig. 5.

To examine the combined effect of APD restitution properties and $I_{\mathrm{Na}}$ recovery dynamics on the occurrence of electrical instability in tissue, we combined the four different APD restitution parameter settings with the three different $I_{\mathrm{Na}}$ dynamics discussed above. This resulted in a total of 12 different model settings. Using these settings, we studied the stability of wave propagation in a ring, which is a one-dimensional model for reentry along a fixed path. Note that it was shown in Ref. 6 that the conditions for instability in a ring are the same as the conditions for spiral breakup in two dimensions.

In Fig. 6, we show APD as a function of period in the 12 different model settings for a wave circulating on a ring. Figure $6 A$ shows restitution for parameter setting $1\left(S_{\max }=0.7\right)$ combined with either the standard, intermediate, or LR $I_{\mathrm{Na}}$ dynamics (curves are superimposed). We can see that for none of these settings alternans occurs. The $I_{\mathrm{Na}}$ dynamics, however, do affect the period for which block occurs: for standard $I_{\mathrm{Na}}$ dynamics, block occurs for periods $<216 \mathrm{~ms}$; for intermediate dynamics, block occurs for periods $<202 \mathrm{~ms}$; and for LR dynamics, block occurs for periods $<170 \mathrm{~ms}$. In Fig. $6 B$ we can see that for setting $2\left(S_{\max }=1.1\right)$ for standard and intermediate $I_{\mathrm{Na}}$ dynamics, no alternans occurs, but for LR $I_{\mathrm{Na}}$ dynamics, alternans occurs for periods between 255 and 170 $\mathrm{ms}$, with a maximum amplitude of $25 \mathrm{~ms}$.

In Fig. $6 C$, we see that for setting $3\left(S_{\max }=1.4\right)$, no alternans occurs for standard $I_{\mathrm{Na}}$ dynamics. Both the intermediate and LR dynamics result in alternans. For intermediate $I_{\mathrm{Na}}$ dynamics, alternans occurs for periods $<280 \mathrm{~ms}$, with a maximum amplitude of $\sim 50 \mathrm{~ms}$; for LR $I_{\mathrm{Na}}$ dynamics, alternans occurs for periods $<300 \mathrm{~ms}$, with a maximum amplitude of $\sim 90$ ms. Finally, in Fig. $6 D$ we see that for parameter setting $4\left(S_{\max }=1.9\right)$, alternans occurs in all three cases. For the standard dynamics, alternans occurs for periods $<321 \mathrm{~ms}$, and for the intermediate and LR settings for periods $<330 \mathrm{~ms}$. Maximum alternans amplitude increased from around 75 to $125 \mathrm{~ms}$ and to $150 \mathrm{~ms}$ when going from standard to intermediate to LR $\mathrm{I}_{\mathrm{Na}}$ dynamics.

Our results show that for fast (LR) $I_{\mathrm{Na}}$ dynamics, alternans instability occurs for the same APD restitution slopes in a single cell as in a ring of tissue. For slow (standard) and intermediate $I_{\mathrm{Na}}$ dynamics, this is not the case. For standard and intermediate $I_{\mathrm{Na}}$ dynamics, a steeper APD restitution slope is required in a ring than in a single cell to generate alternans. These results suggest that faster sodium recovery dynamics lead to earlier occurrence and larger amplitude of alternans and that slower sodium recovery suppresses alternans.

Spiral wave dynamics and alternans in a plane. In Fig. 7 we show snapshots of spiral wave dynamics for the 12 different model settings that we studied in the previous section. The three columns represent the three different $I_{\mathrm{Na}}$ dynamics (standard, intermediate, LR). The four rows represent the four different parameter settings resulting in the four different APD restitution slopes. If spiral breakup occurs, the time of first wave break is indicated in the top right corner. Spiral wave dynamics were simulated for $4 \mathrm{~s}$. If no spiral breakup occurred in this time frame, spiral wave dynamics were assumed to be stable.
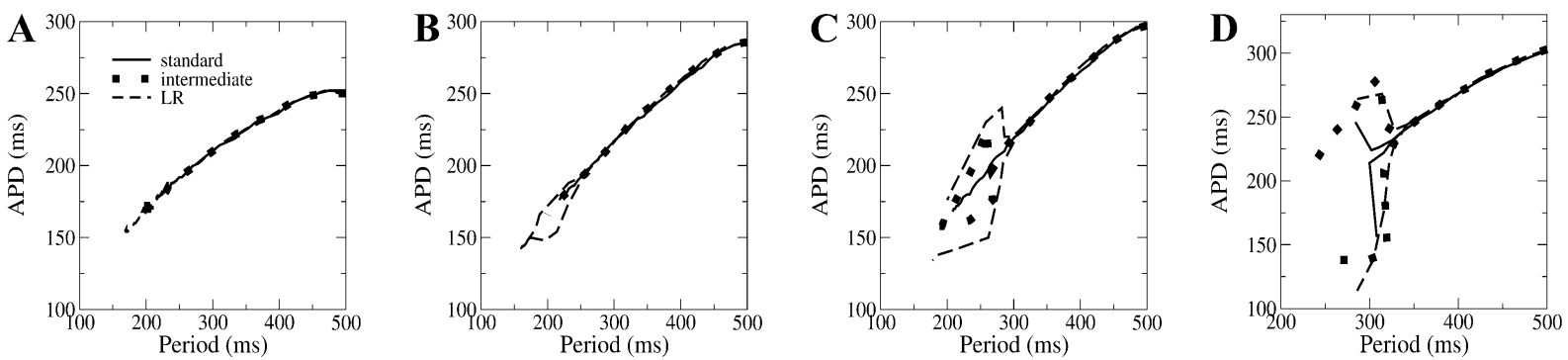

Fig. 6. APD restitution in a ring for the 12 different model settings. Restitution curves are obtained by initiating a pulse propagating in one direction that propagates until steady-state APD is reached, after which the ring length and hence BCL is shortened. $A$ : restitution for the model setting with a maximum slope of 0.7 combined with the standard, intermediate, and Luo-Rudy (33) model (LR) fast $I_{\mathrm{Na}}$ dynamics. $B$ : restitution for the model setting with a maximum slope of 1.1 combined with the standard, intermediate, and LR fast $I_{\mathrm{Na}}$ dynamics. $C$ : restitution for the model setting with a maximum slope of 1.4 combined with the standard, intermediate, and LR fast $I_{\mathrm{Na}}$ dynamics. $D$ : restitution for the model setting with a maximum slope of 1.9 combined with the standard, intermediate, and LR fast $I_{\mathrm{Na}}$ dynamics. 

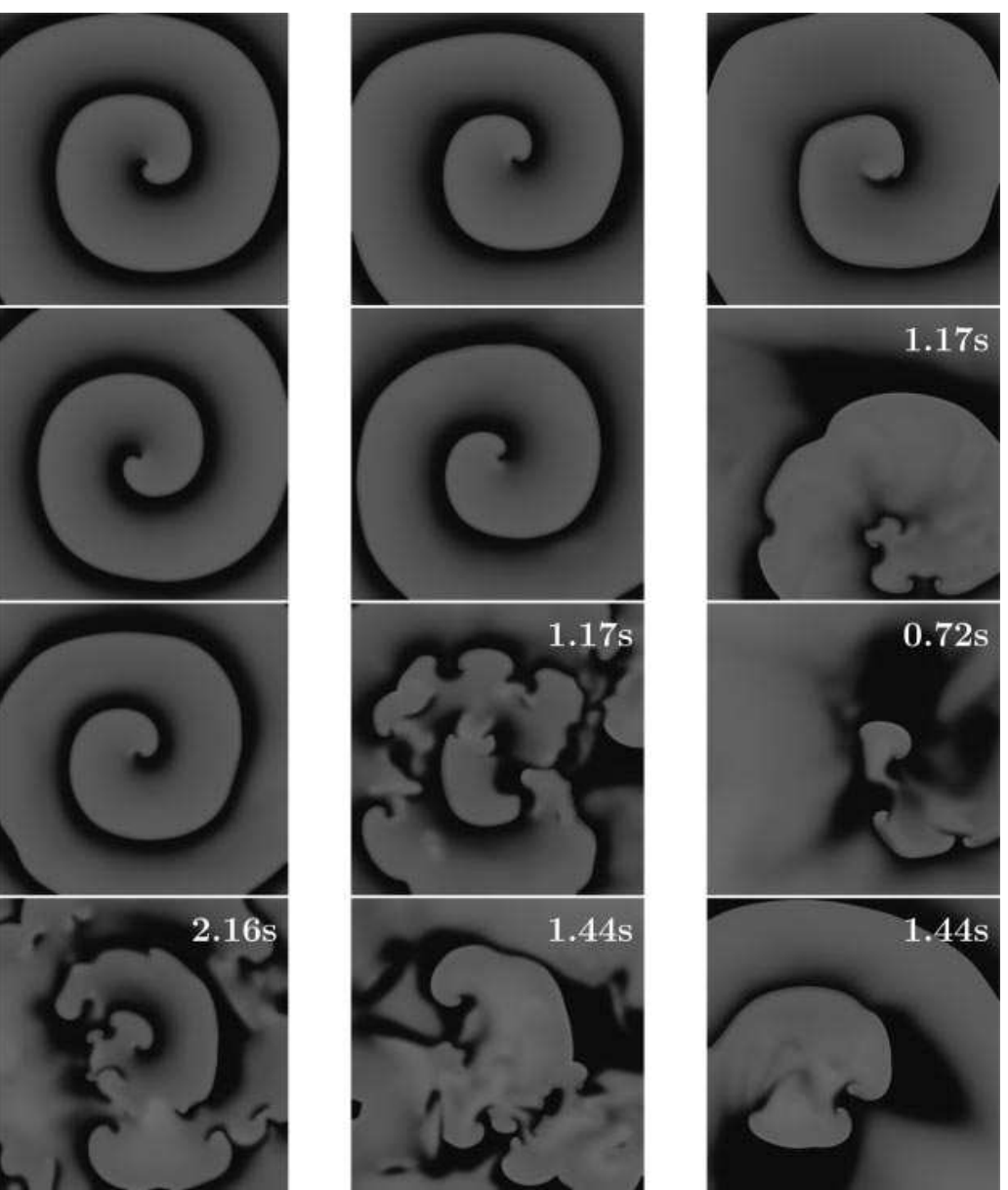

Fig. 7. Snapshots of spiral wave dynamics for the 12 different model settings. Columns (left to right): column 1, standard $I_{\mathrm{Na}}$ dynamics; column 2, intermediate $I_{\mathrm{Na}}$ dynamics; column 3, LR $I_{\mathrm{Na}}$ dynamics. Rows (top to bottom): row 1, slope 0.7 setting;

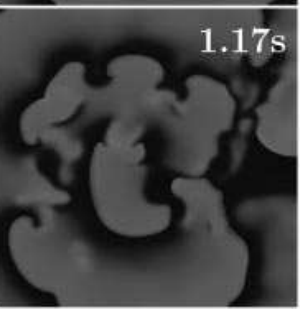

$0.72 \mathrm{~s}$ row 2, slope 1.1 setting; row 3, slope 1.4 setting; row 4, slope 1.9 setting. If spiral breakup occurs, the time of first wave break is indicated at top right corner.

We can see that for parameter setting $1\left(S_{\max }=0.7\right.$, first row), stable spiral wave dynamics occur for all three $I_{\mathrm{Na}}$ dynamics. For setting $2\left(S_{\max }=1.1\right.$, second row), spiral wave dynamics are stable for the standard and intermediate sodium dynamics, but for the LR dynamics, spiral breakup occurs after $1.17 \mathrm{~s}$. For parameter setting $3\left(S_{\max }=1.4\right.$, third row), spiral wave dynamics are only stable for the standard $I_{N a}$ dynamics. For the intermediate dynamics, spiral breakup occurs after $2 \mathrm{~s}$, while for the LR dynamics, breakup occurs after $0.72 \mathrm{~s}$. For setting $4\left(S_{\max }=1.9\right.$, fourth row $)$, spiral breakup occurs for all three $I_{\mathrm{Na}}$ dynamics. For the standard sodium dynamics, breakup occurs after $2.16 \mathrm{~s}$; for the other two sodium dynamics, breakup occurs after $1.44 \mathrm{~s}$.

The conditions we find for spiral breakup in two dimensions correspond nicely with the conditions we find for alternans in a ring. Using some extra parameter settings resulting in restitution slopes in between the 1.4 and 1.9 we used here, we found that the occurrence of alternans/spiral breakup under standard sodium recovery required a minimum APD restitution slope of 1.5 (data not shown).

\section{How Do $I_{N a}$ Dynamics Affect Instability?}

From the previous sections we conclude that for LR $I_{\mathrm{Na}}$ dynamics in our model a slope just over 1 (1.1) suffices to generate alternans and spiral breakup, whereas for the standard $I_{\mathrm{Na}}$ dynamics in our model, a slope of 1.5 is required to generate alternans and spiral breakup. This implies that slow $I_{\mathrm{Na}}$ recovery dynamics suppress alternans instability and breakup. Figure 8 shows the effect of $I_{\mathrm{Na}}$ dynamics on two factors that have been shown to be important for alternans instability: CV restitution and the DIs visited during spiral wave rotation.

Figure $8 A$ shows three $\mathrm{CV}$ restitution curves generated with our model for the three different $I_{\mathrm{Na}}$ dynamics. We can see that the standard $I_{\mathrm{Na}}$ dynamics results in a CV restitution curve that
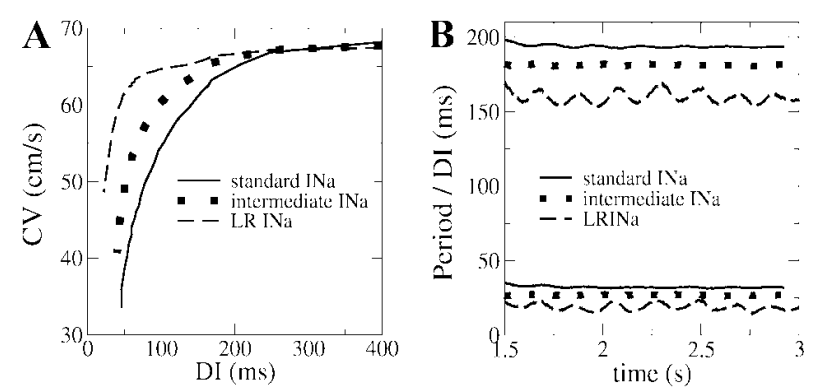

Fig. 8. A: conduction velocity $(\mathrm{CV})$ restitution for the shallow parameter setting of our model (slope 0.7 ) combined with the standard, intermediate, and LR fast $I_{\mathrm{Na}}$ dynamics. Restitution was measured in a cable of 800 cells. $B$ : period (3 top lines) and DI (3 bottom lines) as a function of time, for spiral wave rotation for the shallow parameter setting of our model combined with the standard, intermediate, and LR fast $I_{\mathrm{Na}}$ dynamics. 
Table 3. Dependence of spiral wave period and DI on $I_{N a}$ recovery dynamics and size

\begin{tabular}{cccr}
\hline \hline$I_{\mathrm{Na}}$ Recovery Dynamics & $G_{\mathrm{Na}}$ & Period & DI \\
\hline Standard & Normal & 194 & 32 \\
LR & Normal & 168 & 19 \\
Standard & $\times 7$ & 184 & 22 \\
LR & $\times 0.4$ & 196 & 29 \\
\hline
\end{tabular}

Spiral wave simulations were performed for the parameter settings with a slope $0.7 / 0.8$ to ensure stable spiral wave rotation for all $I_{\mathrm{Na}}$ dynamics. $I_{\mathrm{Na}}$ dynamics are either the standard dynamics of our human ventricular cell model or the Luo-Rudy model (33) (LR) dynamics. $G_{\mathrm{Na}}$ is either as for the default parameter settings of our model (Table 1) or multiplied by the indicated factor. Period and diastolic interval (DI) are mean values and are in ms.

starts gradually declining for DIs of $250 \mathrm{~ms}$ and smaller. The LR $I_{\mathrm{Na}}$ dynamics result in a CV restitution curve that is nearly flat for a broad range of DIs and then declines fast for DIs $<65$ ms. We can see that the intermediate $I_{\mathrm{Na}}$ dynamics indeed result in an intermediate $\mathrm{CV}$ restitution curve. It has been shown in both analytical and modeling studies that a shallow $\mathrm{CV}$ restitution curve suppresses alternans and spiral breakup $(6,7,10)$ : in tissue, through electrotonic interactions, gradual $\mathrm{CV}$ restitution increases the threshold value for APD restitution slopes above which instability occurs above 1 .

Figure $8 B$ shows periods and DIs visited during spiral wave rotation for the three different $I_{\mathrm{Na}}$ dynamics. For illustration purposes, we did this for parameter setting 1 (restitution slope $<1)$ to ensure stable spiral wave rotation for all sodium dynamics. We can see that when going from standard to intermediate to LR sodium dynamics, both period and DI decrease. It is a well-known effect that slowing of spiral wave rotation leads to stabilization. We found in our earlier studies that the mechanism behind this stabilization is that for slower sodium recovery and hence longer periods and DIs, a less steep part of the restitution curve is visited, thus suppressing alternans and spiral breakup $(44,62,63)$.

Is it the shallower CV restitution or the longer DI during spiral wave rotation, or both, that leads to suppression of instability? We tried to resolve this issue by changing DI while keeping the shape of the $\mathrm{CV}$ restitution curve the same. This can be achieved by changing maximal conductance $\left(G_{\mathrm{Na}}\right)$ of the sodium current. Table 3 shows period and DIs of spiral wave rotation for parameter setting 1, either our standard or LR $I_{\mathrm{Na}}$ dynamics and different $G_{\mathrm{Na}}$. From Table 3 we can see that DI can be reduced from $32 \mathrm{~ms}$, occurring for standard $I_{\mathrm{Na}}$ dynamics, to $22 \mathrm{~ms}$, close to the DI for LR $I_{N a}$ dynamics, by increasing $G_{\mathrm{Na}}$ by a factor of 7 . Similarly, we can increase DI from $19 \mathrm{~ms}$, occurring for the LR $I_{\mathrm{Na}}$ dynamics, to $29 \mathrm{~ms}$, close to the DI for standard $I_{\mathrm{Na}}$ dynamics, by multiplying $G_{\mathrm{Na}}$ with a factor of 0.4. Note that although the $\mathrm{CV}$ restitution curve will shift as a result of these modifications, its shape will be maintained (not shown). Furthermore, we assume that multiplication of $G_{\mathrm{Na}}$ by these factors will also work for the other parameter settings of our model.

We simulated spiral wave dynamics for parameter settings 2 and 3 (slope 1.1 and 1.4, respectively) either using our standard $I_{\mathrm{Na}}$ dynamics combined with $G_{\mathrm{Na}} \times 7$ or using LR $I_{\mathrm{Na}}$ dynamics combined with $G_{\mathrm{Na}} \times 0.4$. We see (Fig. $9 B$ ) that for setting 2 only increasing DI for LR $I_{N a}$ dynamics suffices to remove spiral breakup; thus we obtain the same spiral wave dynamics as for our standard $I_{\mathrm{Na}}$ dynamics. Similarly, Fig. $9 C$ shows that for setting 3 , decreasing DI for standard $I_{\mathrm{Na}}$ dynamics induces spiral breakup, thus resulting in the same spiral wave dynamics as for LR $I_{\mathrm{Na}}$ dynamics. These two simulations thus suggest that changes in DI alone can explain the effects of the different $I_{\mathrm{Na}}$ dynamics on alternans and spiral wave stability observed in this study. However, our other simulation results do not support this conclusion. For setting 2, decreasing DI for standard $I_{\mathrm{Na}}$ dynamics does not result in the onset of spiral breakup, as observed for LR $I_{N a}$ dynamics; however, it does result in transient initial wave breaks (Fig. 9A). For setting 3, increasing DI for LR $I_{\mathrm{Na}}$ does not prevent spiral breakup (Fig. 9D), as expected from computations for standard $I_{\mathrm{Na}}$ dynamics. Therefore, we conclude that although the DIs visited during spiral wave rotation have a pronounced effect on the onset of alternans and spiral breakup, CV restitution also plays an important role in determining whether instability will occur.

\section{DISCUSSION}

\section{New Version of Human Ventricular Cell Model}

In this study we developed a new version of our human ventricular cell model. In the new model we incorporated a subspace calcium variable that controls the dynamics of the $I_{\mathrm{CaL}}$ and the ryanodine receptor current. Similar approaches have been taken in many recent models $(19,20,54)$. In addition, we changed the gating dynamics of $I_{\mathrm{CaL}}$, which now has a fast subspace calcium inactivation gate $f_{\text {cass }}$, and a slow and fast voltage inactivation gate $f$ and $f_{2}$, resulting in $I_{\mathrm{CaL}}$ dynamics that agree better with experimental data. We have also replaced the phenomenological description of CICR in our previous model with a reduced version of the ryanodine receptor Markov model developed by Stern et al. (57) and Shannon et al. (54). We demonstrated that with this new description our
A

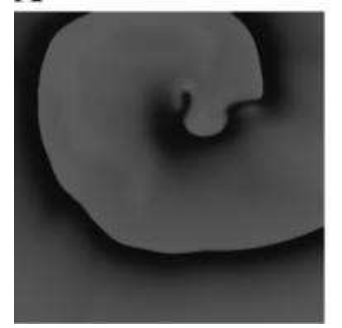

B

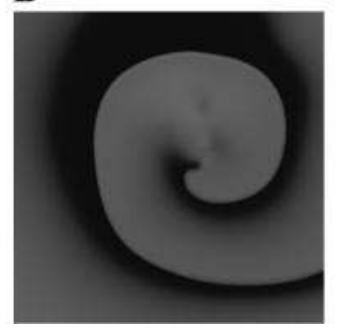

C

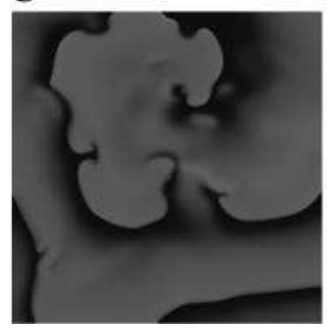

D

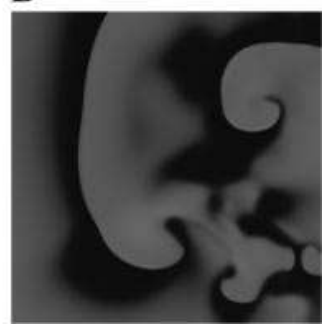

Fig. 9. Snapshots of spiral wave dynamics for 4 different model settings. $A$ : slope 1.1 , standard $I_{\mathrm{Na}}$ dynamics $G_{\mathrm{Na}} \times 7$. $B:$ slope 1.1 , LR $I_{\mathrm{Na}}$ dynamics $G_{\mathrm{Na}} \times$ 0.4. $C$ : slope 1.4 , standard $I_{\mathrm{Na}}$ dynamics $G_{\mathrm{Na}} \times 7$. D: slope 1.4 , LR $I_{\mathrm{Na}}$ dynamics $G_{\mathrm{Na}} \times 0.4$. 
model is able to reproduce a nonlinear gain function for calcium release as a function of SR calcium load.

Although we improved the description of calcium handling in this new version of our model, our formulation is still a simplification and cannot describe all details of calcium dynamics. For example, to describe gradedness of CICR, much more complex models incorporating the description of individual L-type channels, ryanodine receptors, etc., are necessary $(15,51)$. However, such models are computationally very demanding and therefore not feasible for modeling cardiac tissue sheets or complete ventricles, for which our model is intended.

\section{Conditions for Alternans and Spiral Breakup}

We use our new model to study the conditions under which electrical instability and spiral breakup occur to establish whether the spiral breakup hypothesis of VF can be valid for human ventricular tissue. We focus both on the role of APD restitution, reproducing a representative range of recently measured human ventricular restitution curves from Nash et al. (39), and on the role of the recovery dynamics of the fast $I_{\mathrm{Na}}$, of which we found in our previous study that it is much slower than was previously assumed in a lot of cardiac tissue modeling studies (61).

We find that for fast (LR) $I_{\mathrm{Na}}$ recovery dynamics, an APD restitution slope just over 1 (1.1) is enough to result in alternans in single cells and rings of cells and to generate spiral breakup in two-dimensional sheets of tissue. For slower (standard and intermediate) $I_{\mathrm{Na}}$ recovery dynamics, steeper APD slopes are required to get electrical instability in rings and sheets of tissue.

These findings imply that slow $I_{\mathrm{Na}}$ recovery dynamics have a protective effect against steep APD restitution-mediated instability and spiral breakup. This may be an important finding, given that alternans and breakup are most often studied in models with fast (LR) $I_{\mathrm{Na}}$ dynamics. As we demonstrated earlier (61), voltage-clamp data for human cardiac fast sodium channels show slow $I_{\mathrm{Na}}$ recovery dynamics. Here we find that for the standard $I_{\mathrm{Na}}$ dynamics of our model, which are based on these experimental data, an APD restitution slope significantly steeper than 1 , for our particular settings 1.5 , is needed to generate spiral breakup. This 1.5 is well within the range of experimentally found values $(38-40,43)$. Therefore, our results do support that steep APD restitution-mediated instability is a potential mechanism for VF in the human heart.

\section{Mechanism of Breakup Suppression}

Slow $I_{N a}$ dynamics lead both to longer periods and DIs of spiral wave rotation (Fig. $8 B$ ) and a more gradual CV restitution curve (Fig. 8A). We performed a qualitative study of the relative importance of DI during spiral wave rotation and more gradual CV restitution for the suppression of instability, by isolating the $\mathrm{CV}$ effect and compensating for the DI effect of the different $I_{\mathrm{Na}}$ dynamics using conductance. We found that in some cases the DI effect can explain differences in spiral breakup or no spiral breakup for the different $I_{\mathrm{Na}}$ dynamics. However, for other parameter settings, differences in spiral wave stability could not be explained merely by the DI effect. This suggests that spiral wave period is not the only important effect of slower $I_{\mathrm{Na}}$ recovery that leads to alternans suppression and that the $\mathrm{CV}$ effect also plays a role in suppressing instability. Unfortunately, we found no way to change CV restitution shape while keeping the DI of spiral wave rotation constant to further establish the precise importance of CV restitution vs. DI.

\section{Relation to Work of Others}

In analytical studies, it was shown that for waves propagating in tissue, the condition for steep restitution-mediated alternans instability was not simply slope $>1$ but is slope $>1+$ $\xi c^{\prime} / c^{2}(7,10)$. Here $\xi$ represents the strength of action potential morphology (especially repolarization wave back)-dependent electrotonic interactions, $c^{\prime}$ is the derivative of the CV curve for the relevant DI (i.e., that of rotation on a ring or spiral wave rotation), and $c$ is the maximum planar $\mathrm{CV}$. From the formula it follows that the larger the value of $\xi$ (stronger electrotonic interactions) and the larger the value of $c^{\prime} / c^{2}$ (large change of $\mathrm{CV}$ over range of DIs, or low $\mathrm{CV}$ ), the steeper is the slope required to generate instability. Here we indeed found that the slower the $I_{\mathrm{Na}}$ recovery dynamics, and hence the more gradual the $\mathrm{CV}$ restitution curve, the steeper was the APD restitution slope needed to generate instability. Our results agree with a previous study from $\mathrm{Qu}$ et al. (48), who showed that a CV restitution curve that stays flat over a broad range of DIs, similar to our steep CV curve, promotes spiral breakup, and to results from Fox et al. (13), who showed that chances of conduction block decrease for lower $\mathrm{CVs}$ at short cycle lengths, corresponding to our more gradual CV restitution. Our results also agree with results from an extensive simulation study on the dependence of alternans and spiral breakup on APD restitution, $\mathrm{CV}$ restitution, electrotonic interactions, and cardiac memory performed by Cherry and Fenton (6). They found that for action potentials with a fast repolarizing wave back, leading to strong electrotonic interactions, a more gradual CV restitution can suppress alternans in a ring and spiral breakup in two dimensions. Note that in our human ventricular cell model, we have a strong repolarizing wave back caused by the large inward rectifier $\mathrm{K}^{+}$current $\left(I_{\mathrm{K} 1}\right)$.

Our results may seem dissimilar from results such as those of Cao et al. (5) and Watanabe et al. (65), who showed that a more gradual CV restitution is proarrhythmic because it promotes the spatial discordance of alternans, causing spiral breakup to require a smaller tissue size. However, as noted by Cherry and Fenton (6), gradual CV restitution has a dual effect: on the one hand it can suppress alternans provided that electrotonic interactions are strong, and on the other hand it promotes the spatial discordance of any alternans still present. Whether alternans arises thus depends on the strength of electrotonic interactions, the shape of $\mathrm{CV}$ restitution, and tissue size. In our model, we found in the two-dimensional sheets of tissue of $20 \times 20 \mathrm{~cm}$ only the alternans-suppressing effect. This suggests that a larger tissue size is needed for discordant alternans to develop. Given that $20 \times 20 \mathrm{~cm}$ is large relative to human heart size, we conclude that for the human ventricles and standard $I_{\mathrm{Na}}$ dynamics, the alternans-suppressing effect will dominate.

The results we found here also agree with previous work from our group $(44,62,63)$. In these studies we found that the presence of inexcitable obstacles or the removal of gap junctional connections between cells leads to suppression of spiral 
breakup. This suppression was caused by the slowing down of spiral wave rotation, leading to longer DIs, similar to the effect of slower fast $I_{\mathrm{Na}}$ recovery. Longer DIs shift the position of the spiral wave on the (unchanged) restitution curve to the right and upward, away from the steepest part.

\section{Limitations}

The ionic mechanisms responsible for the variation in APD restitution curves are currently unknown. In this study, to obtain different restitution slopes, we used more general knowledge of the influence of certain parameters on APD and APD restitution. We varied the dynamics of $I_{\mathrm{CaL}}$, as it has been shown in both modeling studies $(12,49)$ and experiments $(50)$ that $I_{\mathrm{CaL}}$ is a major determinant of restitution slope. APD was then readjusted by varying conductances of $I_{\mathrm{Kr}}, I_{\mathrm{Ks}}, I_{\mathrm{pCa}}$, and $I_{\mathrm{pK}}$ (Table 2). If new data on the ionic current differences underlying restitution variation become available, new parameter settings based on these data need to be constructed. However, we expect that restitution slope rather than the parameter setting is important for the observed effects, and hence that results will stay similar.

In this study we find that a slow $I_{N a}$ recovery dynamics protect against steep APD restitution-mediated alternans. However, we investigated this in homogeneous rings and sheets of cardiac tissue, whereas real cardiac tissue is heterogeneous in numerous properties, including APD and APD restitution (39, 43), is anisotropic, and has a complex anatomy. What the precise conditions for spiral breakup are under more realistic conditions therefore remains to be investigated. In addition, we did not investigate the influence of the amount of electrotonic coupling and cardiac memory on the conditions for alternans and spiral breakup.

Furthermore, the steep APD restitution we studied here is not the only mechanism that can lead to alternans instability. Other instabilities, for example in the intracellular calcium dynamics $(47,55,56)$, can lead to action potential alternans. Finally, other mechanisms than alternans leading to spiral breakup have been suggested to underlie VF, for example, the mother rotor hypothesis.

In conclusion, we propose a novel model for human ventricular cells. We report that $I_{\mathrm{Na}}$ recovery dynamics play an important role in the occurrence of steep restitution-mediated dynamical electrical instability. The slow recovery dynamics found in experiments on human cardiac $I_{\mathrm{Na}}$ suppress electrical instability. We conclude that steep restitution-mediated fibrillation can occur in human ventricular tissue. However, an APD restitution slope considerably steeper than 1 is required.

\section{APPENDIX}

No changes were made to formulations of the following currents: $I_{\mathrm{Na}}, I_{\mathrm{to}}, I_{\mathrm{Kr}}, I_{\mathrm{K} 1}, I_{\mathrm{NaCa}}, I_{\mathrm{NaK}}, I_{\mathrm{pCa}}, I_{\mathrm{pK}}, I_{\mathrm{bNa}}$, and $I_{\mathrm{bCa}}$. For these formulations, we refer to their description in the previous version of our model (61).

\section{L-Type $\mathrm{Ca}^{2+}$ Current}

$$
\begin{gathered}
I_{\mathrm{CaL}}=G_{\mathrm{CaL}} d f f_{2} f_{\mathrm{cass}} 4 \frac{(V-15) F^{2}}{R T} \frac{0.25 \mathrm{Ca}_{\mathrm{Ss}} e^{2(V-15) F / R T}-\mathrm{Ca}_{\mathrm{o}}}{e^{2(V-15) F / R T}-1} \\
d_{\infty}=\frac{1}{1+e^{(-8-V) / 7.5}}
\end{gathered}
$$

$$
\begin{aligned}
\alpha_{d} & =\frac{1.4}{1+e^{(-35-V) / 13}}+0.25 \\
\beta_{d} & =\frac{1.4}{1+e^{(V+5) / 5}} \\
\gamma_{d} & =\frac{1}{1+e^{(50-V) / 20}} \\
\tau_{d} & =\alpha_{d} \beta_{d}+\gamma_{d} \\
f_{\infty} & =\frac{1}{1+e^{(V+20) / 7}} \\
\alpha_{f} & \left.=1102.5 e^{-\left(\frac{V+27}{15}\right.}\right)^{2} \\
\beta_{f} & =\frac{200}{1+e^{(13-V) / 10}} \\
\gamma_{f} & =\frac{180}{1+e^{(V+30) / 10}}+20 \\
\tau_{f} & =\alpha_{f}+\beta_{f}+\gamma_{f} \\
\tau_{f \text { cass }} & =\frac{0.67}{1+\left(\frac{\mathrm{Ca}}{0.05}\right)^{2}}+2 \\
\tau_{f 2} & =\alpha_{f 2}+\beta_{f 2}+\gamma_{f 2} \\
\gamma_{\text {cass } \infty} & =\frac{0.6}{1+e^{(V+35) / 7}}+0.33 \\
\alpha_{f 2} & =600 e^{-\frac{(V+25)^{2}}{170}} \\
& =\frac{31}{1+e^{(25-V) / 10}} \\
& \left.=\frac{16}{0.05}\right)^{(V+30) / 10} \\
&
\end{aligned}
$$

Slow Delayed Rectifier Current

$$
\begin{aligned}
& I_{\mathrm{Ks}}=G_{\mathrm{Ks}} x_{\mathrm{s}}^{2}\left(V-E_{\mathrm{Ks}}\right) \\
& x_{\mathrm{s} \infty}=\frac{1}{1+e^{(-5-V) / 14}} \\
& \alpha_{x \mathrm{~s}}=\frac{1400}{\sqrt{1+e^{(5-V) / 6}}} \\
& \beta_{x \mathrm{~s}}=\frac{1}{1+e^{(V-35) / 15}} \\
& \tau_{x \mathrm{~s}}=\alpha_{x s} \beta_{x \mathrm{~s}}+80
\end{aligned}
$$

\section{Calcium Dynamics}

In the following equations, $\mathrm{Ca}_{\text {itotal }}$ is total (free + buffered) cytoplasmic $\mathrm{Ca}^{2+}$ concentration; $\mathrm{Ca}_{\mathrm{SR} \text { total }}$ is total $\mathrm{SR} \mathrm{Ca}{ }^{2+}$ concentration; $\mathrm{Ca}_{\text {sStotal }}$ is total diadic subspace $\mathrm{Ca}^{2+}$ concentration; $\mathrm{Ca}_{\mathrm{i}}$ is free cytoplasmic $\mathrm{Ca}^{2+}$ concentration; $\mathrm{Ca}_{\mathrm{SR}}$ is free $\mathrm{SR} \mathrm{Ca}^{2+}$ concentration; Cass is free diadic subspace $\mathrm{Ca}^{2+}$ concentration; $I_{\text {rel }}$ is CICR current; $I_{\text {up }}$ is SR Ca ${ }^{2+}$ pump current; $I_{\text {leak }}$ is $\mathrm{SR} \mathrm{Ca}^{2+}$ leak current; $I_{\text {xfer }}$ is diffusive $\mathrm{Ca}^{2+}$ current between diadic $\mathrm{Ca}^{2+}$ subspace and bulk 
cytoplasm; $\mathrm{O}$ is proportion of open $I_{\text {rel }}$ channels; and $\overline{\mathrm{R}}$ is proportion of closed $I_{\text {rel }}$ channels (for parameters, see Table 1).

$$
\begin{aligned}
& I_{\text {leak }}=V_{\text {leak }}\left(\mathrm{Ca}_{\mathrm{SR}}-\mathrm{Ca}_{\mathrm{i}}\right) \\
& I_{\text {up }}=\frac{V_{\text {maxup }}}{1+K_{\text {up }}^{2} / \mathrm{Ca}_{\mathrm{i}}^{2}} \\
& I_{\text {rel }}=V_{\text {rel }} \mathrm{O}\left(\mathrm{Ca}_{\mathrm{SR}}-\mathrm{Ca}_{\mathrm{SS}}\right) \\
& I_{\text {xfer }}=V_{\text {xfer }}\left(\mathrm{Ca}_{\mathrm{SS}}-\mathrm{Ca}_{\mathrm{i}}\right) \\
& \mathrm{O}=\frac{k_{1} \mathrm{Ca}_{\mathrm{SS}}^{2} \overline{\mathrm{R}}}{k_{3}+k_{1} \mathrm{Ca}_{\mathrm{SS}}^{2}} \\
& \frac{\mathrm{d} \overline{\mathrm{R}}}{\mathrm{d} t}=-k_{2} \mathrm{Ca}_{\mathrm{SS}} \overline{\mathrm{R}}+k_{4}(1-\overline{\mathrm{R}}) \\
& k_{1}=\frac{k_{1^{\prime}}}{k_{\text {casr }}} \\
& k_{2}=k_{2}, k_{\text {casr }} \\
& k_{\text {casr }}=\max _{\mathrm{sr}}-\frac{\max _{\mathrm{sr}}-\min _{\mathrm{sr}}}{1+\left(\mathrm{EC} / \mathrm{Ca}_{\mathrm{SR}}\right)^{2}} \\
& \mathrm{Ca}_{\text {ibufc }}=\frac{\mathrm{Ca}_{\mathrm{i}} \times \mathrm{Buf}_{\mathrm{c}}}{\mathrm{Ca}_{\mathrm{i}}+K_{\text {bufc }}} \\
& \mathrm{dCa}_{\mathrm{itotal}} / \mathrm{d} t=-\frac{I_{\mathrm{bCa}}+I_{\mathrm{pCa}}-2 I_{\mathrm{NaCa}}}{2 \mathrm{~V}_{\mathrm{c}} \mathrm{F}}+\frac{\mathrm{V}_{\mathrm{sr}}}{\mathrm{V}_{\mathrm{c}}}\left(I_{\text {leak }}-I_{\text {up }}\right)+I_{\text {xfer }} \\
& \mathrm{Ca}_{\text {srbufsr }}=\frac{\mathrm{Ca}_{\text {sr }} \times \mathrm{Buf}_{\text {sr }}}{\mathrm{Ca}_{\text {sr }}+K_{\text {bufs }}} \\
& \mathrm{dCa}_{\text {SRtotal }} / \mathrm{d} t=\left(I_{\text {up }}-I_{\text {leak }}-I_{\text {rel }}\right) \\
& \mathrm{Ca}_{\text {ssbufss }}=\frac{\mathrm{Ca}_{\mathrm{ss}} \times \mathrm{Buf}_{\mathrm{ss}}}{\mathrm{Ca}_{\mathrm{ss}}+\mathrm{K}_{\text {bufss }}} \\
& \mathrm{dCa}_{\mathrm{SStotal}} / \mathrm{d} t=-\frac{I_{\mathrm{CaL}}}{2 V_{\mathrm{SS}} \mathrm{F}}+\frac{\mathrm{V}_{\mathrm{sr}}}{\mathrm{V}_{\mathrm{ss}}} I_{\text {rel }}-\frac{\mathrm{V}_{\mathrm{c}}}{\mathrm{V}_{\mathrm{ss}}} I_{\text {xfer }}
\end{aligned}
$$

\section{ACKNOWLEDGMENTS}

We thank Dr. J. Sneyd, Dr. M. Nash, and Dr. P. Taggart for valuable discussions.

\section{GRANTS}

This work was supported by the Netherlands Organization for Scientific Research through Grant 635100004 of the Research Council for Physical Sciences (K. H. W. J. ten Tusscher).

\section{REFERENCES}

1. Banville I, Chattipakorn N, and Gray RA. Restitution dynamics during pacing and arrhythmias in isolated pig hearts. $J$ Cardiovasc Electrophysiol 15: 455-463, 2004

2. Banville I and Gray RA. Effect of action potential duration and conduction velocity restitution and their spatial dispersion on alternans and the stability of arrhythmias. J Cardiovasc Electrophysiol 13: 1141-1149, 2002.

3. Benitah J, Bailly P, D'Agrosa M, Da Ponte J, Delgado C, and Lorente P. Slow inward current in single cells isolated from adult human ventricles. Pflügers Arch 421: 176-187, 1992.

4. Beuckelmann DJ, Nabauer M, and Erdmann E. Characteristics of calcium-current in isolated human ventricular myocytes from patients with terminal heart failure. J Mol Cell Cardiol 23: 929-937, 1991.

5. Cao J, Qu Z, Kim Y, Wu T, Garfinkel A, Weiss JN, Karagueuzian HS, and Chen P. Spatiotemporal heterogeneity in the induction of ventricular fibrillation by rapid pacing, importance of cardiac restitution properties. Circ Res 84: 1318-1331, 1999.

6. Cherry EM and Fenton FH. Suppression of alternans and conduction blocks despite steep APD restitution: electrotonic, memory and conduc- tion velocity effects. Am J Physiol Heart Circ Physiol 286: H2332-H2341, 2004

7. Cytrynbaum E and Keener JP. Stability conditions for the traveling pulse: modifying the restitution hypothesis. Chaos 12: 788-799, 2002.

8. Drouin E, Charpentier F, Gauthier C, Laurent $\mathbf{K}$, and Le Marec $\mathbf{H}$. Electrophysiologic characteristics of cells spanning the left ventricular wall of human heart: evidence for the presence of M cells. J Am Coll Cardiol 26: 185-192, 1995.

9. Drouin E, Lande G, and Charpentier F. Amiodarone reduces transmural heterogeneity of repolarization in the human heart. J Am Coll Cardiol 32: 1063-1067, 1998.

10. Echebarria B and Karma A. Instability and spatiotemporal dynamics of alternans in paced cardiac tissue. Phys Rev Lett 88: 208101, 2002.

11. Fox JJ, Bodenschatz E, and Gilmour RF Jr. Period-doubling instability and memory in cardiac tissue. Phys Rev Lett 89: 138101, 2002.

12. Foz JJ, McHarg JL, and Gilmour RF. Ionic mechanism of electrical alternans. Am J Physiol Heart Circ Physiol 282: H516-H530, 2002.

13. Fox JJ, Riccio ML, Drury P, Werthman A, and Gilmour RF Jr. Dynamic mechanism for conduction block in heart tissue. New J Phys 5: 101.1-101.14, 2003.

14. Gilmour RF, Heger JJ, Prystowsky EN, and Zipes DZ. Cellular electrophysiologic abnormalities of diseased human ventricular myocardium. Am J Cardiol 51: 137-144, 1983.

15. Greenstein JL and Winslow RL. An integrative model of the cardiac ventricular myocyte incorporating local control of $\mathrm{Ca}^{2+}$ release. Biophys J 83: 2918-2945, 2002.

16. Guevara MR, Ward A, Shrier A, and Glass L. Electrical alternans and period doubling bifurcations. IEEE Comp Cardiol 562: 167-170, 1984.

17. Haws CW and Lux RL. Correlation between in vivo transmembrane action potential durations and activation-recovery intervals from electrograms. Effects of interventions that alter repolarization time. Circulation 81: 281-288, 1990.

18. Hayashi H, Miyauchi Y, Chou CC, Karagueuzian HS, Chen PS, and Lin SF. Effects of cytochalasin D on electrical restitution and the dynamics of ventricular fibrillation in isolated rabbit heart. J Cardiovasc Electrophysiol 14: 1077-1084, 2003.

19. Hund TJ and Rudy Y. Rate dependence and regulation of action potential and calcium transient in a canine cardiac ventricular cell model. Circulation 110: 3168-3174, 2004.

20. Iyer V, Mazhari R, and Winslow RL. A computational model of the human left-ventricular epicardial myocyte. Biophys $J$ 87: 1507-1525, 2004.

21. Karma A. Spiral breakup in model equations of action potential propagation in cardiac tissue. Phys Rev Lett 71: 1103-1106, 1993.

22. Karma A. Electrical alternans and spiral wave breakup in cardiac tissue. Chaos 4: 461-472, 1994.

23. Keener J and Sneyd J. Mathematical Physiology. New York: SpringerVerlag, 1998.

24. Koller ML, Maier SK, Gelzer AR, Bauer WR, Meesmann M, and Gilmour RF Jr. Altered dynamics of action potential restitution and alternans in humans with structural heart disease. Circulation 112: 1542 1548, 2005.

25. Koller ML, Riccio ML, and Gilmour RF Jr. Dynamic restitution of action potential duration during electrical alternans and ventricular fibrillation. Am J Physiol Heart Circ Physiol 275: H1635-H1642, 1998.

26. Koller ML, Riccio ML, and Gilmour RF Jr. Effects of $\left[\mathrm{K}^{+}\right]_{\mathrm{o}}$ on electrical restitution and activation dynamics during ventricular fibrillation. Am J Physiol Heart Circ Physiol 279: H2665-H2672, 2000.

27. Lee MH, Lin SF, Ohara T, Omichi C, Okuyama Y, Chudin E, Garfinkel A, Weiss JN, Karagueuzian HS, and Chen PS. Effects of diacetyl monoxime and cytochalasin $\mathrm{D}$ on ventricular fibrillation in swine right ventricles. Am J Physiol Heart Circ Physiol 280: H2689-H2696, 2001.

28. Li G, Lau C, Leung T, and Nattel S. Ionic current abnormalities associated with prolonged action potentials in cardiomyocytes from diseased human right ventricles. Heart Rhythm 4: 460-468, 2004.

29. Li G, Yang B, Feng J, Bosch RF, Carrier M, and Nattel S. Transmembrane $I_{\mathrm{Ca}}$ contributes to rate-dependent changes of action potentials in human ventricular myocytes. Am J Physiol Heart Circ Physiol 276: H98-H106, 1999.

30. Li GR, Feng J, Yue L, and Carrier M. Transmural heterogeneity of action potentials and $I_{\text {tol }}$ in myocytes isolated from the human right ventricle. Am J Physiol Heart Circ Physiol 275: H369-H377, 1998. 
31. Li GR, Feng J, Yue L, Carrier M, and Nattel S. Evidence for two components of delayed rectifier $\mathrm{K}^{+}$current in human ventricular myocytes. Circ Res 78: 689-696, 1996.

32. Li GR and Nattel S. Properties of human atrial $I_{\mathrm{Ca}}$ at physiological temperatures and relevance to action potential. Am J Physiol Heart Circ Physiol 272: H227-H235, 1997.

33. Luo C and Rudy Y. A model of the ventricular cardiac action potential, depolarization, repolarization, and their interaction. Circ Res 68: 15011526, 1991.

34. Magyar J, Iost N, Kortvely A, Banyasz T, Virag L, Szigligeti P, Varro A, Opincariu M, Szecsi J, Papp JG, and Nanasi PP. Effects of endothelin-1 on calcium and potassium currents in undiseased human ventricular myocytes. Pflïgers Arch 441: 144-149, 2000.

35. Magyar J, Szentandrassy N, Banyasz T, Fulop L, Varro A, and Nanasi PP. Effects of thymol on calcium and potassium currents in canine and human ventricular cardiomyocytes. Br J Pharmacol 136: 330-338, 2002.

36. Mewes T and Ravens U. L-type calcium currents of human myocytes from ventricle of non-failing and failing hearts and atrium. $J$ Mol Cell Cardiol 26: 1307-1320, 1994.

37. Morgan JM, Cunningham D, and Rowland E. Dispersion of monophasic action potential duration: demonstrable in humans after premature ventricular extrastimulation but not in steady state. J Am Coll Cardiol 19: 1244-1253, 1992.

38. Nash MP, Bradley CP, Sutton P, Hayward M, Paterson DJ, and Taggart P. Human hearts possess large regions of steep and flat APD restitution. Europace 6, Suppl 1: 187, 2004.

39. Nash MP, Bradley CP, Sutton PM, Clayton RH, Kallis P, Hayward M, Paterson DJ, and Taggart P. Whole heart APD restitution properties in cardiac patients: A combined clinical and modeling study. Exp Physiol g1: $33 \mathrm{~g}-35 \mathrm{~g}, 2006$

40. Nash MP, Bradley CP, Sutton PM, Hayward M, Paterson DJ, and Taggart P. Spatial heterogeneity of action potential duration restitution in humans. Heart Rhythm 2, Suppl 1: S216-S217, 2005.

41. Nolasco JB and Dahlen RW. A graphic method for the study of alternation in cardiac action potentials. J Appl Physiol 25: 191-196, 1968

42. Otani NF and Gilmour RF Jr. Memory models for the electrical properties of local cardiac systems. J Theor Biol 187: 409-436, 1997.

43. Pak HN, Hong SJ, Hwang GS, Lee HS, Park SW, Ahn JC, Moo Ro Y, and Kim YH. Spatial dispersion of action potential duration restitution kinetics is associated with induction of ventricular tachycardia/fibrillation in humans. J Cardiovasc Electrophysiol 15: 1357-1363, 2004.

44. Panfilov AV. Spiral breakup in an array of coupled cells: the role of the intercellular conductance. Phys Rev Lett 88: 118101-1-118101-4, 2002

45. Panfilov AV and Holden AV. Self-generation of turbulent vortices in a two-dimensional model of cardiac tissue. Phys Lett A 147: 463-466, 1990.

46. Pelzmann B, Schaffer P, Bernhart E, Lang P, Machler H, Rigler B, and Koidl B. L-type calcium current in human ventricular myocytes at a physiological temperature from children with tetralogy of Fallot. Cardiovasc Res 38: 424-432, 1998.

47. Pruvot EJ, Katra RP, Rosenbaum DS, and Laurita KR. Role of calcium cycling versus restitution in the mechanism of repolarization alternans. Circ Res 94: 1083-1090, 2004.

48. Qu Z, Weiss JN, and Garfinkel A. Cardiac electrical restitution properties and stability of reentrant spiral waves: a simulation study. Am J Physiol Heart Circ Physiol 276: H269-H283, 1999.
49. Qu Z, Xie F, Garfinkel A, and Weiss JN. Origins of spiral wave meander and breakup in a two-dimensional cardiac tissue model. Ann Biomed Eng 28: 755-771, 2000.

50. Riccio ML, Koller ML, and Gilmour RF Jr. Electrical restitution and spatiotemporal organization during ventricular fibrillation. Circ Res 84: 955-963, 1999.

51. Rice JJ, Jafri MS, and Winslow RL. Modeling gain and gradedness of $\mathrm{Ca}^{2+}$ release in the functional unit of the cardiac diadic space. Biophys $J$ 77: 1871-1884, 1999.

52. Rush $\mathbf{S}$ and Larsen $\mathbf{H}$. A practical algorithm for solving dynamic membrane equations. IEEE Trans Biomed Eng 25: 389-392, 1978.

53. Shannon TR, Ginsburg KS, and Bers DM. Potentiation of fractional sarcoplasmic reticulum calcium release by total and free intra-sarcoplasmic reticulum calcium concentration. Biophys J 78: 334-343, 2000.

54. Shannon TR, Wang F, Puglisi J, Weber C, and Bers DM. A mathematical treatment of integrated $\mathrm{Ca}$ dynamics within the ventricular myocyte. Biophys J 87: 3351-3371, 2004.

55. Shiferaw Y, Watanabe MA, Garfinkel A, Weiss JN, and Karma A. Model of intracellular calcium cycling in ventricular myocytes. Biophys $J$ 85: 3666-3686, 2003

56. Shimizu W and Antzelevitch C. Cellular and ionic basis for T-wave alternans under long-QT conditions. Circulation 99: 1499-1507, 1999.

57. Stern MD, Song LS, Cheng H, Sham JS, Yang HT, Boheler KR, and Rios E. Local control models of cardiac excitation-contraction coupling. A possible role for allosteric interactions between ryanodine receptors. $J$ Gen Physiol 113: 469-489, 1999.

58. Sun H, Leblanc N, and Nattel S. Mechanisms of inactivation of L-type calcium channels in human atrial myocytes. Am J Physiol Heart Circ Physiol 272: H1625-H1635, 1997.

59. Taggart $\mathbf{P}$, Sutton $\mathbf{P}$, Chalabi Z, Boyett MR, Simon R, Elliott D, and Gill JS. Effect of adrenergic stimulation on action potential duration restitution in humans. Circ Res 33: 54-62, 1973.

60. Taggart P, Sutton PMI, Opthof T, Coronel R, Trimlett R, Pugsley W, and Kallis $\mathbf{P}$. Inhomogeneous transmural conduction during early ischemia in patients with coronary artery disease. J Mol Cell Cardiol 32: 621-639, 2000.

61. Ten Tusscher KHWJ, Noble D, Noble PJ, and Panfilov AV. A model for human ventricular tissue. Am J Physiol Heart Circ Physiol 286: H1573-H1589, 2004.

62. Ten Tusscher KHWJ and Panfilov AV. Influence of nonexcitable cells on spiral breakup in two-dimensional and three-dimensional excitable media. Phys Rev E 68: 062902-1-062902-4, 2003.

63. Ten Tusscher KHWJ and Panfilov AV. Wave propagation in excitable media with randomly distributed obstacles. Multiscale Model Simul 3: 265-282, 2005.

64. Tolkacheva EG, Schaeffer DG, Gauthier DJ, and Krassowska W. Condition for alternans and stability of the 1:1 response pattern in a "memory" model of paced cardiac dynamics. Phys Rev E Stat Nonlin Soft Matter Phys 67: 031904, 2003.

65. Watanabe MA, Fenton FH, Evans SJ, Hastings HM, and Karma A. Mechanisms for Discordant Alternans. J Cardiovasc Electrophysiol 12: 196-206, 2001.

66. Wu T, Lin S, Weiss JN, Ting C, and Chen P. Two types of ventricular fibrillation in isolated rabbit hearts. Circulation 106: 1859-1866, 2002.

67. Yue AM, Franz MR, Robers PR, and Morgan JM. Global endocardial electrical restitution in human right and left ventricles determined by noncontact mapping. Am J Cardiol 46: 1067-1075, 2005. 